\title{
Secure Massive MIMO System with Two-Way Relay Cooperative Transmission in $6 \mathrm{G}$ Networks
}

\author{
Yumeng Su \\ Harbin Engineering University \\ Hongyuan Gao ( $\nabla$ gaohongyuan@hrbeu.edu.cn ) \\ Harbin Engineering University https://orcid.org/0000-0001-8505-6828 \\ Shibo Zhang \\ Harbin Engineering University
}

\section{Research}

Keywords: 6G, physical-layer security, massive MIMO, multiple-relay collaboration, two-way relaying

Posted Date: May 27th, 2020

DOI: https://doi.org/10.21203/rs.3.rs-30336/v1

License: (1) This work is licensed under a Creative Commons Attribution 4.0 International License.

Read Full License 


\section{Secure Massive MIMO System with Two-Way Relay \\ 2 Cooperative Transmission in 6G Networks}

3 Yumeng Su, Hongyuan Gao*, and Shibo Zhang

4 College of Information and Communication Engineering, Harbin Engineering University, Harbin

$5 \quad 150001$, China

6 Abstract With the advent of Internet of Everything (IoE) and the era of big data, massive multiple-input multiple-output (MIMO) is considered an essential technology to meet the growing communication requirements for beyond $5 \mathrm{G}$ and the forthcoming 6G networks. This paper considers a secure massive MIMO system, where the legitimate user and the base station (BS) exchange messages via two-way relays with the presence of passive eavesdroppers. To achieve the trade-off between the physical-layer security and communication reliability, we design a cooperative transmission mode based on multiple-relay collaboration, where some relays broadcast the received signals and other relays act as friendly jammers to prevent the interception by eavesdroppers. A quantum chemical reaction optimization (QCRO) algorithm is proposed to find the most suitable scheme for multiple-relay collaboration. Simulation results highlight excellent performance of the proposed transmission mode under QCRO in different communication scenarios, which can be considered a potential solution for the security issue in future wireless networks.

Keywords: 6G, physical-layer security, massive MIMO, multiple-relay collaboration, two-way relaying

\footnotetext{
${ }^{*}$ Corresponding author: Hongyuan Gao
} E-mail: gaohongyuan@hrbeu.edu.cn 


\section{Introduction}

In contemporary society, as the booming development of information technology and the popularity of intelligent equipment, wireless networks have become an essential part of our daily life [1]. Inheriting the benefits achieved in 5G, 6G network is expected to expand to a wider level to realize the full coverage of land and air [2-5]. As networks become denser, how to efficiently utilize the system resources and how to meet the higher transmission demands of ultra-high speed, high quality, and low latency have becoming key topics in 6G networks [3-5]. Massive MIMO is an effective solution for the increasing challenge of wireless data traffic since it can serve a large number of IoT devices at the same time [6-9]. By utilizing large antenna arrays, massive MIMO system can transmit several directional beams to the desired terminals, which can greatly improve the quality of service (QoS) and spectral efficiency of communication systems [10].

Due to the openness of transmission environments and the broadcast nature of wireless channels, secure transmission has becoming a critical issue in wireless networks $[11,12]$. Since eavesdroppers will make every effort to intercept or break the information transmission, many scholars have conducted in-depth research on how to protect the transmission of confidential information. Typically, cryptographic encryption method was used to maintain the system privacy [13-15]. However, the implementation complexity is too high due to the cumbersome process of service management. By exploiting wireless transmission properties, physical-layer security has attracted considerable attentions in massive MIMO networks [16, 17]. Several efforts such as artificial noise [18-20], antenna/relay selection [21-23], and cooperative strategies [24-27] have been employed to deal with the information leakage by eavesdroppers. The utilization of antenna correlation diversity was 
demonstrated to improve the secrecy rate of massive MIMO systems [19]. With the assistance of cooperative relays, the authors in [24] and [25] investigated the secure transmission problem in the presence of passive and active eavesdroppers. Hoang et al. [26] investigated the secrecy performance under a practical scenario where the eavesdroppers hide their existence in the system. In [21], Kuhestani et al. considered the scenario in a cooperative massive MIMO relay network where untrusted relays act as potential eavesdroppers. A destination-based cooperative jamming is implemented to improve the system capacity and transmission confidentiality.

Among recent works on the security issue, the combinations of cooperative relays and secure techniques have shown significant improvements on secrecy capacity and system coverage. Due to the low-complexity relaying process and high spectral efficiency outperforms one-way relay network, two-way relays have attracted extensive attentions [28-31]. In two-way relay networks, eavesdroppers receive the overlapped information when the two legitimate devices broadcast their signals at the same time [31]. In this condition, both two devices act as source and jammer to prevent the interception by eavesdroppers. By exploiting the dual property, the authors in [32] and [33] investigated distributed relay selection criterion to optimize the system security performance. In order to enhance the spatial diversity gains and further improve the system performance, massive MIMO with two-way relay collaboration has become a hotspot in recent researches [34-37]. In [35], Feng et al. indicated that the system performance with full-duplex operation outperforms the half-duplex mode under a certain self-loop interference. Zhang et al. [36] investigated the overall performance with hardware impairments. In [37], Pan et al. adopted a power allocation strategy based on geometric programming to reduce the information interception of untrusted users. These works, however, did not consider the 
73 information leakage happens at the two phases of data transmission.

\subsection{Motivation and contributions}

Table 1 Comparison with existing works

\begin{tabular}{llccccc}
\hline Reference & Category & $\begin{array}{c}\text { Power } \\
\text { allocation }\end{array}$ & $\begin{array}{c}\text { Security } \\
\text { strategy }\end{array}$ & $\begin{array}{c}\text { Cooperative } \\
\text { communication }\end{array}$ & $\begin{array}{c}\text { Multiple-relay } \\
\text { selection }\end{array}$ & $\begin{array}{c}\text { Using } \\
\text { duality }\end{array}$ \\
\hline [16]-[20] & DT & $\checkmark$ & $\checkmark$ & $\times$ & $\times$ & $\times$ \\
{$[21]$} & OWR & $\checkmark$ & $\checkmark$ & $\checkmark$ & $\times$ & $\times$ \\
{$[22]$} & OWR & $\times$ & $\times$ & $\checkmark$ & $\times$ & $\times$ \\
{$[23]$} & OWR & $\checkmark$ & $\checkmark$ & $\checkmark$ & $\checkmark$ & $\times$ \\
{$[24]$} & OWR & $\checkmark$ & $\times$ & $\checkmark$ & $\times$ & $\times$ \\
{$[25]-[27]$} & OWR & $\checkmark$ & $\checkmark$ & $\checkmark$ & $\times$ & $\times$ \\
{$[28]$} & TWR & $\times$ & $\times$ & $\checkmark$ & $\times$ & $\times$ \\
{$[29]$} & TWR & $\checkmark$ & $\times$ & $\checkmark$ & $\times$ & $\times$ \\
{$[30]$} & TWR & $\checkmark$ & $\checkmark$ & $\checkmark$ & $\times$ & $\checkmark$ \\
{$[31]$} & TWR & $\times$ & $\checkmark$ & $\checkmark$ & $\times$ & $\times$ \\
{$[32,33]$} & TWR & $\times$ & $\times$ & $\checkmark$ & $\times$ & $\checkmark$ \\
{$[37]$} & TWR & $\checkmark$ & $\times$ & $\checkmark$ & $\times$ & $\checkmark$ \\
Our work & TWR & $\checkmark$ & $\checkmark$ & $\checkmark$ & $\checkmark$ & $\checkmark$ \\
\hline
\end{tabular}

(DT: Direct transmission OWR: One-way relaying TWR: Two-way relaying)

Although existing researches have made influential achievements in improving the security performance, there are still some limitations. For better understanding, the comparison of previous works with our work is presented in Table 1. Many anti-eavesdropping methods [16-20] generally consider the case of direct transmission between the BS and the users, but it's hard to meet the QoS demands of the desired terminals where there are no direct transmission links due to the long-distance fading. For secure transmission issues with relay collaboration, most of the existing works [21-33, 37] do not simultaneously address the power allocation, resource utilization, and multiple relay selection in a scenario where information leakage happens at both transmission phases in massive MIMO networks with limited time-frequency resources. In this paper, we present a cooperative transmission mode for the security 
issue of massive MIMO two-way relay networks. Unlike one-way relaying systems, both the two legitimate devices play the roles of source and jammer via two-way relaying operation. However, the duality has not been well exploited in $[28,29,31]$ to prevent the interception by eavesdroppers. In order to break through the limitations of previous studies and obtain a higher security performance, we propose a multiple-relay collaboration strategy considering the interception of eavesdroppers during the two phases of information transmission. According to the multiple-relay selection (MRS) scheme, the relays function as receivers/transmitters or friendly jammers to reduce the information leakage between the source and the legitimate user. Considering the energy conservation and communication reliability requirements, we propose a quantum chemical reaction optimization (QCRO) algorithm to obtain the optimal MRS result. The major contributions of our work are summarized in the following:

- We present a low-complexity cooperative transmission mode to balance the communication security and reliability of a massive MIMO two-way relay system. A multiple-relay collaboration strategy is employed to enhance the secrecy performance while prevent the interception by eavesdroppers in two transmission phases.

- Exact expression for secrecy sum-rate is derived considering the information leakage of two transmission phases in massive MIMO relay networks. The expression indicates that secrecy performance is affected by the transmission power of relays and MRS result with QoS and interference constraints in practical scenarios.

- A novel algorithm named QCRO is proposed to tackle the complicated MRS problem for the cooperative transmission in massive MIMO networks. 
Simulation results illustrate the excellent performance of QCRO over conventional algorithms in various system parameters. Besides, the proposed QCRO algorithm can be considered as a potential solution for other complicated problems in communication domain.

\subsection{Organization and notations}

The other sections of this work are presented as follows. Section 2 presents the system architecture and the analysis of a secure massive MIMO network with multiple-relay collaboration. The QCRO algorithm for MRS is addressed in Section 3, and Section 4 presents the simulations. In the end, we conclude this work in the final section.

Notations: The uppercase boldface symbols and lowercase boldface symbols represent matrixes and the vectors, respectively. $\mathbf{0}$ and $\boldsymbol{I}_{N}$ represent the zero vector and identity matrix, respectively. $\mathrm{CN}\left(\mu, \sigma^{2}\right)$ denotes the complex Gaussian distribution with mean of $\mu$ and variance of $\sigma^{2} .(.)^{\mathrm{T}}$ and $(.)^{\mathrm{H}}$ represent transpose operator and conjugate transpose operator, respectively. |.|, $\|$.$\| , and abs(.) represent$ modulus, Euclidean norm, and absolute value functions, respectively. $E\{$.$\} denotes$ the statistical expectation, and $[x]^{+}$stands for $\max \{0, x\}$.

\section{System model}

In this paper, we introduce a secure massive MIMO system where there is a BS with $M_{t}$ antennas, a user, $L$ relays, and $K$ eavesdroppers. The BS and the user exchange their information via two-way relay networks, and each eavesdropper tries to intercept the desired signals during the two phases of transmission. Regarding propagation model, the channel state information (CSI) is defined as $\boldsymbol{g}_{X, Y}=\boldsymbol{h}_{X, Y} d_{X, Y}^{-\xi / 2}$ 
[26], where $\boldsymbol{h}_{X, Y} \sim \mathrm{CN}\left(\mathbf{0}, \boldsymbol{I}_{N}\right)$ denotes the small-scale fading factor, and $d_{X, Y}^{-\xi / 2}$ denotes the large-scale fading factor. In it, $\xi$ denotes the path loss exponent, and $d_{X, Y}$ denotes the distance between the $\mathrm{X}-\mathrm{Y}$ link. The global CSI is available by uplink training in time-division duplexed (TDD) massive MIMO networks [11, 38]. We consider the instantaneous CSI remains unchanged in one time slot. The definitions of CSI between the terminals of secure massive MIMO two-way relay networks are as follows:

- CSI from the BS to the $i$-th relay: $\boldsymbol{g}_{B S, r_{i}}$

- CSI from the BS to the $k$-th eavesdropper: $\boldsymbol{g}_{B S, e_{k}}$

- CSI from the user to the $k$-th eavesdropper: $g_{u, e_{k}}$

- CSI from the $i$-th relay to the user: $g_{r_{i}, u}$

- CSI from the $i$-th relay to the $k$-th eavesdropper: $g_{r_{i}, e_{k}}$

- CSI from the $i$-th relay to the $j$-th $\operatorname{relay~}(i \neq j): g_{r_{i}, r_{j}}$

In the following, we introduce the cooperative transmission mode with cooperative relaying and cooperative jamming (CRCJ) for the secure massive MIMO two-way relay network.

\subsection{CRCJ policy}

Since the direct transmission link between the BS and the user is so weak due to the long-distance fading, we consider a scenario where the BS and the user exchange their desired signals via two-way relays. In this system, the BS and the user broadcast their signals with transmit power $p_{B S}$ and $p_{u}$. Under CRCJ policy, some relays are selected to receive the mixed signal from the BS and the user, while the remaining relays act as jammers to transmit jamming signals to prevent the information 
interception by eavesdroppers. For simplicity, the MRS scheme is expressed by a binary vector $\boldsymbol{b}=\left[b_{1}, b_{2}, \ldots, b_{L}\right]$, where $b_{i} \in\{0,1\}, i=1,2, \ldots, L$. If $b_{i}=1$, the $i$-th relay is selected to assist information transmission between the legitimate devices. The signal received at the $i$-th relay is given by

$$
y_{i}=\sqrt{p_{B S}} \boldsymbol{g}_{B S, r_{i}} \boldsymbol{V} \boldsymbol{s}_{B S}+\sqrt{p_{u}} g_{r_{i}, u} s_{u}+\sum_{j=1}^{L}\left(1-b_{j}\right) \sqrt{p_{r_{j}}} g_{r_{i}, r_{j}} s_{r_{j}}+\eta_{r_{i}}
$$

where $p_{r_{j}}$ is the transmit power of the $j$-th jammer; $V$ is the precoding matrix at the BS; $s_{B S}, s_{u}$, and $s_{r_{j}}$ are unit-power signals with $E\left\{\left\|s_{B S}\right\|^{2}\right\}=1, E\left\{\left|s_{u}\right|^{2}\right\}=1$, and $E\left\{\left|s_{r_{j}}\right|^{2}\right\}=1$, respectively; $\eta_{r_{i}}$ is the additive white Gaussian noise (AWGN).

For the $k$-th eavesdropper, the intercepted signal in the first phase can be expressed as

$$
y_{e_{k}}^{(1)}=\sqrt{p_{B S}} \boldsymbol{g}_{B S, e_{k}} \boldsymbol{V} \boldsymbol{s}_{B S}+\sqrt{p_{u}} g_{u, e_{k}} s_{u}+\sum_{j=1}^{L}\left(1-b_{j}\right) \sqrt{p_{r_{j}}} g_{r_{j}, e_{k}} s_{r_{j}}+\eta_{e_{k}}^{(1)}
$$

where $\eta_{e_{k}}^{(1)}$ is the AWGN. The signal-to-interference plus noise ratio (SINR) received at the $k$-th eavesdropper from the two legitimate devices (BS and user) can be respectively given by

$$
\begin{gathered}
\gamma_{B S \rightarrow e_{k}}^{(1)}=\frac{p_{B S}\left\|\boldsymbol{g}_{B S, e_{k}} \boldsymbol{V}\right\|^{2}}{p_{u}\left|g_{u, e_{k}}\right|^{2}+\sum_{j=1}^{L}\left(1-b_{j}\right) p_{r_{j}}\left|g_{r_{j}, e_{k}}\right|^{2}+\sigma_{0}^{2}} \\
\gamma_{u \rightarrow e_{k}}^{(1)}=\frac{p_{u}\left|g_{u, e_{k}}\right|^{2}}{p_{B S}\left\|\boldsymbol{g}_{B S, e_{k}} \boldsymbol{V}\right\|^{2}+\sum_{j=1}^{L}\left(1-b_{j}\right) p_{r_{j}}\left|g_{r_{j}, e_{k}}\right|^{2}+\sigma_{0}^{2}}
\end{gathered}
$$

175 where $\sigma_{0}^{2}$ denotes the power of AWGN.

In the second phase of data transmission, the selected relay $i$ broadcast the 
normalized signal to the legitimate devices with the transmit power of $p_{i}$. The normalized transmission signal is denoted as

$$
y_{i}^{\text {norm }}=\sqrt{G_{i}} y_{i}=\frac{y_{i}}{\sqrt{p_{B S}\left\|g_{B S, r_{i}} \boldsymbol{V}\right\|^{2}+p_{u}\left|g_{r_{i}, u}\right|^{2}+\sum_{j=1}^{L}\left(1-b_{j}\right) p_{r_{j}}\left|g_{r_{i}, r_{j}}\right|^{2}+\sigma_{0}^{2}}}
$$

$180 \quad$ where $\quad G_{i}=\left(p_{B S}\left\|\boldsymbol{g}_{B S, r_{i}} \boldsymbol{V}\right\|^{2}+p_{u}\left|g_{r_{i}, u}\right|^{2}+\sum_{j=1}^{L}\left(1-b_{j}\right) p_{r_{j}}\left|g_{r_{i}, r_{j}}\right|^{2}+\sigma_{0}^{2}\right)^{-1} \quad$ is $\quad$ the normalization factor. After self-interference cancellation (SIC), the corresponding signals at the $\mathrm{BS}$ and the user can be respectively expressed by

$$
\boldsymbol{y}_{B S}=\sum_{i=1}^{L} b_{i} \sqrt{G_{i} p_{i}} \boldsymbol{W} \boldsymbol{g}_{B S, r_{i}}^{\mathrm{T}} \cdot\left(\sqrt{p_{u}} g_{r_{i}, u} s_{u}+\sum_{j=1}^{L}\left(1-b_{j}\right) \sqrt{p_{r_{j}}} g_{r_{i}, r_{j}} s_{r_{j}}+\eta_{r_{i}}\right)+\boldsymbol{\eta}_{B S}
$$

and

$$
y_{u}=\sum_{i=1}^{L} b_{i} \sqrt{G_{i} p_{i}} g_{r_{i}, u} \cdot\left(\sqrt{p_{B S}} \boldsymbol{g}_{B S, r_{i}} \boldsymbol{V s}_{B S}+\sum_{j=1}^{L}\left(1-b_{j}\right) \sqrt{p_{r_{j}}} g_{r_{i}, r_{j}} s_{r_{j}}+\eta_{r_{i}}\right)+\eta_{u}
$$
the BS and the user are respectively shown by

$$
\gamma_{u \rightarrow B S}=\frac{\sum_{i=1}^{L} b_{i} G_{i} p_{i} p_{u}\left\|\boldsymbol{W g}_{B S, r_{i}}^{\mathrm{T}}\right\|^{2} \cdot\left|g_{r_{i}, u}\right|^{2}}{\sum_{i=1}^{L}\left[b_{i} G_{i} p_{i}\left\|\boldsymbol{W} \boldsymbol{g}_{B S, r_{i}}^{\mathrm{T}}\right\|^{2} \cdot\left(\sum_{j=1}^{L}\left(1-b_{j}\right) p_{r_{j}}\left|g_{r_{i}, r_{j}}\right|^{2}+\sigma_{0}^{2}\right)\right]+\sigma_{0}^{2}}
$$

189 and

190

$$
\gamma_{B S \rightarrow u}=\frac{\sum_{i=1}^{L} b_{i} G_{i} p_{i} p_{B S}\left|g_{r_{i}, u}\right|^{2} \cdot\left\|\boldsymbol{g}_{B S, r_{i}} \boldsymbol{V}\right\|^{2}}{\sum_{i=1}^{L}\left[b_{i} G_{i} p_{i}\left|g_{r_{i}, u}\right|^{2} \cdot\left(\sum_{j=1}^{L}\left(1-b_{j}\right) p_{r_{j}}\left|g_{r_{i}, r_{j}}\right|^{2}+\sigma_{0}^{2}\right)\right]+\sigma_{0}^{2}}
$$


given by

$$
C_{B S \rightarrow u}=\frac{1}{2} \log _{2}\left(1+\gamma_{B S \rightarrow u}\right)
$$

and

$$
C_{u \rightarrow B S}=\frac{1}{2} \log _{2}\left(1+\gamma_{u \rightarrow B S}\right)
$$

For the $k$-th eavesdropper, the intercepted signal in the second phase can be given by

199

$$
\begin{aligned}
y_{e_{k}}^{(2)} & =\sum_{i=1}^{L} b_{i} \sqrt{G_{i} p_{i}} g_{r_{i}, e_{k}} y_{i}+\eta_{e_{k}}^{(2)} \\
& =\sum_{i=1}^{L} b_{i} \sqrt{G_{i} p_{i}} g_{r_{i}, e_{k}} \cdot\left(\sqrt{p_{B S}} \boldsymbol{g}_{B S, r_{i}} \boldsymbol{V} \boldsymbol{s}_{B S}+\sqrt{p_{u}} g_{r_{i}, u} s_{u}+\sum_{j=1}^{L}\left(1-b_{j}\right) \sqrt{p_{r_{j}}} g_{r_{i}, r_{j}} s_{r_{j}}+\eta_{r_{i}}\right)+\eta_{e_{k}}^{(2)}
\end{aligned}
$$

200

Assuming that the eavesdroppers are independent of each other, the total received 205 SINR at the passive eavesdroppers from the BS and the user can be respectively given 206

where $\eta_{e_{k}}^{(2)}$ is the AWGN. The SINR received at the $k$-th eavesdropper from the BS and the user can be respectively shown by

$$
\begin{gathered}
\gamma_{B S \rightarrow e_{k}}^{(2)}=\frac{\sum_{i=1}^{L} b_{i} G_{i} p_{i} p_{B S}\left|g_{r_{i}, e_{k}}\right|^{2} \cdot\left\|\boldsymbol{g}_{B S, r_{i}} \boldsymbol{V}\right\|^{2}}{\sum_{i=1}^{L}\left[b_{i} G_{i} p_{i}\left|g_{r_{i}, e_{k}}\right|^{2} \cdot\left(p_{u}\left|g_{r_{i}, u}\right|^{2}+\sum_{j=1}^{L}\left(1-b_{j}\right) p_{r_{j}}\left|g_{r_{i}, r_{j}}\right|^{2}+\sigma_{0}^{2}\right)\right]+\sigma_{0}^{2}} \\
\gamma_{u \rightarrow e_{k}}^{(2)}=\frac{\sum_{i=1}^{L} b_{i} G_{i} p_{i} p_{u}\left|g_{r_{i}, e_{k}}\right|^{2} \cdot\left|g_{r_{i}, u}\right|^{2}}{\sum_{i=1}^{L}\left[b_{i} G_{i} p_{i}\left|g_{r_{i}, e_{k}}\right|^{2} \cdot\left(p_{B S}\left\|\boldsymbol{g}_{B S, r_{i}} \boldsymbol{V}\right\|^{2}+\sum_{j=1}^{L}\left(1-b_{j}\right) p_{r_{j}}\left|g_{r_{i}, r_{j}}\right|^{2}+\sigma_{0}^{2}\right)\right]+\sigma_{0}^{2}}
\end{gathered}
$$




$$
\gamma_{B S}^{\mathrm{E}}=\max _{k \in\{1,2, \ldots, K\}}\left\{\gamma_{B S \rightarrow e_{k}}^{(1)}, \gamma_{B S \rightarrow e_{k}}^{(2)}\right\}
$$

$$
\gamma_{u}^{\mathrm{E}}=\max _{k \in\{1,2, \ldots, K\}}\left\{\gamma_{u \rightarrow e_{k}}^{(1)}, \gamma_{u \rightarrow e_{k}}^{(2)}\right\}
$$

The information leakage from the BS and the user are respectively shown by

$$
C_{B S}^{\mathrm{E}}=\frac{1}{2} \log _{2}\left(1+\gamma_{B S}^{\mathrm{E}}\right)
$$

$$
C_{u}^{\mathrm{E}}=\frac{1}{2} \log _{2}\left(1+\gamma_{u}^{\mathrm{E}}\right)
$$

Hence, the secrecy transmission rates of the BS and the user can be given by

$$
\begin{gathered}
C_{B S \rightarrow u}^{\mathrm{S}}=\left[C_{B S \rightarrow u}-C_{B S}^{\mathrm{E}}\right]^{+} \\
C_{u \rightarrow B S}^{\mathrm{S}}=\left[C_{u \rightarrow B S}-C_{u}^{\mathrm{E}}\right]^{+}
\end{gathered}
$$

Finally, the secrecy sum-rate can be expressed by

$$
C_{\text {sum }}=\left[C_{B S \rightarrow u}-C_{B S}^{E}\right]^{+}+\left[C_{u \rightarrow B S}-C_{u}^{E}\right]^{+}
$$

\subsection{Problem formulation}

Based on the CRCJ policy, the problem of secrecy sum-rate maximization based on MRS is formulated as

$$
\max C_{\text {sum }}(\boldsymbol{b})=\max \left\{\left[C_{B S \rightarrow u}(\boldsymbol{b})-C_{B S}^{E}(\boldsymbol{b})\right]^{+}+\left[C_{u \rightarrow B S}(\boldsymbol{b})-C_{u}^{E}(\boldsymbol{b})\right]^{+}\right\}
$$

subject to

$$
b_{i} \in\{0,1\} \quad \forall i \in\{1,2, \ldots, L\}
$$

$$
\sum_{i=1}^{L} b_{i} \geq 1
$$




$$
\sum_{i=1}^{L}\left[b_{i} G_{i} p_{i}\left|g_{r_{i}, u}\right|^{2} \cdot\left(\sum_{j=1}^{L}\left(1-b_{j}\right) p_{r_{j}}\left|g_{r_{i}, r_{j}}\right|^{2}\right)\right] \leq \text { Interference }
$$

$$
\sum_{i=1}^{L}\left[b_{i} G_{i} p_{i}\left\|\boldsymbol{W g}_{B S, r_{i}}^{\mathrm{T}}\right\|^{2} \cdot\left(\sum_{j=1}^{L}\left(1-b_{j}\right) p_{r_{j}}\left|g_{r_{i}, r_{j}}\right|^{2}\right)\right] \leq \text { Interference }
$$

227 where $p_{\max }$ denotes the maximum transmission power of relays. The relay selection constraints are shown in (22b) and (22c), where (22b) indicates that each relay acts as a jammer or a cooperator to participate in transmission, and (22c) indicates that at least one relay is selected to broadcast confidential signals to the legitimate devices. (22d) denotes the power constraints of cooperative relays and cooperative jammers. (22e) and (22f) are interference constraints of the BS and the user. Due to the receive-and-forward mechanism of two-way relays, the jamming signals transmitted to eavesdroppers can also cause interference to the BS and the legitimate user. Hence, to guarantee the system communication quality, the interference from the cooperative jammers should not exceed the maximum interference threshold $I_{\text {th }}$ of cooperative relays. Hence, the power of the $j$-th cooperative relay is given by

$$
p_{r_{j}}=\min \left\{p_{\max }, \frac{I_{\mathrm{th}}}{L^{\prime}\left|\max _{i \in\{1,2, \ldots, L\}}\left\{\left(1-b_{j}\right) \cdot g_{r_{i}, r_{j}}\right\}\right|^{2}}\right\}
$$

where $L^{\prime}$ denotes the number of cooperative jammers.

Obviously, the optimization problem of (22) is a multi-constraint nonlinear programming problem, and the computational complexity exponentially increases with the number of relays, which is NP-hard to solve. However, traditional algorithms are difficult to get the good performance due to the slow convergence speed and poor convergence accuracy. To efficiently tackle the complicated problem, we propose a 
quantum chemical reaction optimization (QCRO) algorithm to obtain the appropriate solution.

\section{Methods}

In this section, a novel intelligent algorithm named QCRO is proposed for multiple-relay selection in a secure massive MIMO two-way relay network. Inspired by the chemical reaction optimization (CRO) algorithm [39] and quantum evolutionary theory [9], QCRO employs a set of quantum molecules, which is varied by different quantum evolutionary rules. Here we introduce the principle of QCRO.

\subsection{QCRO for optimization problem}

In an $L$-dimensional space, (where $L$ is the maximal dimension of the problem), there exist $N$ quantum molecules. The $n$-th quantum molecule $(n=1,2, \ldots, N)$ of the $t$-th iteration is given by

$$
\boldsymbol{x}_{n}^{t}=\left[x_{n, 1}^{t}, x_{n, 2}^{t}, \ldots, x_{n, L}^{t}\right]
$$

where $0 \leq x_{n, l}^{t} \leq 1 ; n=1,2, \ldots, N ; \quad l=1,2, \ldots, L ; x_{n, l}^{t}$ denotes the $l$-th quantum bit of the $n$-th quantum molecule. For each quantum molecule, the quantum bits should be measured to the solution domain. The measurement state of the $i$-th quantum molecule can be obtained by the following rule:

$$
\bar{x}_{n, l}^{t}= \begin{cases}1, & \alpha_{n, l}^{t}>\left(x_{n, l}^{t}\right)^{2} \\ 0, & \alpha_{n, l}^{t} \leq\left(x_{n, l}^{t}\right)^{2}\end{cases}
$$

where $\bar{x}_{n, l}^{t}$ denotes the $l$-th measurement state of the $i$-th quantum molecule, and $\alpha_{n, l}^{t}$ is a random number distributed in $[0,1]$

The fitness value of the $n$-th quantum molecule is calculated by the fitness 
function, which can be expressed as $f\left(\overline{\boldsymbol{x}}_{n}^{t}\right)$. For the maximum optimization problem, the global optimal solution $\bar{\rho}_{\text {best }}^{t}=\left[\bar{\rho}_{\text {best }, 1}^{t}, \bar{\rho}_{\text {best }, 2}^{t}, \ldots, \bar{\rho}_{\text {best }, L}^{t}\right]$ is denoted as the measurement state of the quantum molecule with the maximum fitness value until the $t$-th iteration.

In QCRO, the quantum molecules are updated by collision, decomposition, and synthesis. These processes are related to the kinetic energy (KE) of quantum molecules, where the top $\mu_{1} N$ quantum molecules with the highest $\mathrm{KE}$ are updated by collision, $\mu_{2} N$ quantum molecules with the smallest KE are updated by synthesis, and the remaining $\mu_{3} N$ quantum molecules are updated by decomposition. $\mu_{1}, \mu_{2}$, and $\mu_{3}$ are constants which respectively represent the reaction ratio of collision, synthesis, and decomposition. To make it easy, we sort the quantum molecules in a descending order according to the level of KE, and the $n^{\prime}$-th quantum molecule is denoted by $\boldsymbol{x}_{n^{\prime}}^{t}=\left[x_{n^{\prime}, 1}^{t}, x_{n^{\prime}, 2}^{t}, \ldots, x_{n^{\prime}, L}^{t}\right]$ with the KE of $e_{n^{\prime}}^{t}$. The generation of new quantum molecules is related to the quantum rotation angle and measurement states of previous quantum molecules. For collision, the $n^{\prime}$-th quantum molecule is updated to a quantum molecule $m$, the quantum rotation angle and $\mathrm{KE}$ are given by

$$
\theta_{m, l}^{t+1}=c_{1} \cdot\left(\bar{x}_{g, l}^{t}-\bar{x}_{n, l}^{t}\right)
$$

where $m=n^{\prime} ; n^{\prime}=1,2, \ldots, \mu_{1} N ; l=1,2, \ldots, L ; \bar{x}_{g, l}^{t}$ denotes the $l$-th measurement state of the $g$-th quantum molecule with higher fitness value in the $t$-th iteration, $g \in\{1,2, \ldots, N\}, g \neq n^{\prime} . \quad c_{1}$ represents the weight coefficient, and Ve represents the loss rate of KE.

For decomposition, the $n^{\prime}$-th quantum molecule is decomposed into quantum 
molecule $m$ and $m+1$, and the quantum rotation angles are respectively shown as

$$
\theta_{m, l}^{t+1}= \begin{cases}c_{2} \cdot\left(\bar{\rho}_{\text {best }, l}^{t}-\bar{x}_{n^{\prime}, l}^{t}\right)+c_{3} \cdot\left(\bar{x}_{a, l}^{t}-\bar{x}_{n^{\prime}, l}^{t}\right), & \varphi_{m, l}^{t+1}<\kappa_{1} \\ c_{4} \cdot\left(\bar{x}_{g, l}^{t}-\bar{x}_{n^{\prime}, l}^{t}\right), & \text { else }\end{cases}
$$

$$
\theta_{m+1, l}^{t+1}= \begin{cases}c_{2} \cdot\left(\bar{\rho}_{\text {best }, l}^{t}-\bar{x}_{n^{\prime}, l}^{t}\right)+c_{3} \cdot\left(\bar{x}_{a, l}^{t}-\bar{x}_{n^{\prime}, l}^{t}\right), & \varphi_{m+1, l}^{t+1}<\kappa_{1} \\ c_{4} \cdot\left(\bar{x}_{g, l}^{t}-\bar{x}_{n^{\prime}, l}^{t}\right), & \text { else }\end{cases}
$$

292 where $m=2 n^{\prime}-\mu_{1} N-1 ; n^{\prime}=\mu_{1} N+1, \mu_{1} N+2, \ldots, \mu_{1} N+\mu_{3} N ; l=1,2, \ldots, L ; g \in\{1,2, \ldots, N\}$, $293 g \neq n^{\prime} ; \bar{x}_{a, l}^{t}$ denotes the $l$-th measurement state of a random quantum molecule in 294 the $t$-th iteration, $a \in\{1,2, \ldots, N\}, a \neq n^{\prime} ; c_{2}, c_{3}$ and $c_{4}$ are weight coefficients. $\kappa_{1}$ is the mutation probability which is a fixed parameter that determines the decomposition style, $\varphi_{m, l}^{t+1}$ and $\varphi_{m+1, l}^{t+1}$ are random variables distributed from 0 to 1 . The KE of quantum molecule $m$ and $m+1$ can be expressed by

$$
e_{m+1}^{t+1}=e_{n^{\prime}}^{t} \cdot(1-\mathrm{V} e) / 2
$$
are synthesized into a new quantum molecule $m$, the quantum rotation angle and $\mathrm{KE}$ are given by

$$
\theta_{m, l}^{t+1}=c_{5} \cdot \omega_{m, l}^{t} \cdot\left(\bar{x}_{n^{\prime}+1, l}^{t}-\bar{x}_{n^{\prime}, l}^{t}\right)
$$

where $\quad m=\left(n^{\prime}+\mu_{1} N+3 \mu_{3} N+1\right) / 2 \quad ; \quad n^{\prime}=\mu_{1} N+\mu_{3} N+1, \mu_{1} N+\mu_{3} N+3, \ldots, N-1 \quad$; $l=1,2, \ldots, L ; \quad c_{5}$ represents the weight coefficient; and $\omega_{m, l}^{t}$ is a random variable distributed from 0 to 1 . 

obtained by

$$
\begin{gathered}
x_{m, l}^{t+1}=\left\{\begin{array}{lr}
\sqrt{1-\left(x_{n^{\prime}, l}^{t}\right)^{2}}, & \theta_{m, l}^{t+1}=0 \text { and } \hat{\varphi}_{m, l}^{t+1} \leq \kappa_{2} \\
\operatorname{abs}\left(x_{n^{\prime}, l}^{t} \cdot \cos \theta_{m, l}^{t+1}-\sqrt{1-\left(x_{n^{\prime}, l}^{t}\right)^{2}} \cdot \sin \theta_{m, l}^{t+1}\right), \text { else }
\end{array}\right. \\
x_{m+1, l}^{t+1}=\left\{\begin{array}{l}
\sqrt{1-\left(x_{n^{\prime}, l}^{t}\right)^{2}}, \\
\operatorname{abs}\left(x_{n^{\prime}, l}^{t} \cdot \cos \theta_{m+1, l}^{t+1}-\sqrt{1-\left(x_{n^{\prime}, l}^{t}\right)^{2}} \cdot \sin \theta_{m+1, l}^{t+1}\right), \text { else }
\end{array}\right.
\end{gathered}
$$

312 where $\hat{\varphi}_{m, l}^{t+1}$ and $\hat{\varphi}_{m+1, l}^{t+1}$ are random variables distributed from 0 to $1, \kappa_{2}$ denotes the

313 conversion probability, and abs(.) represents the absolute value function. For 314 collision, $m=n^{\prime} \quad ; \quad n^{\prime}=1,2, \ldots, \mu_{1} N$. For decomposition, $m=2 n^{\prime}-\mu_{1} N-1$;

$315 n^{\prime}=\mu_{1} N+1, \mu_{1} N+2, \ldots, \mu_{1} N+\mu_{3} N \quad$. For synthesis, $m=\left(n^{\prime}+\mu_{1} N+3 \mu_{3} N+1\right) / 2 \quad ;$ $316 \quad n^{\prime}=\mu_{1} N+\mu_{3} N+1, \mu_{1} N+\mu_{3} N+3, \ldots, N-1$.

317 Then, we obtain the corresponding measurement states of the updated quantum 318 molecules and calculate the fitness value. The measurement state of the quantum 319 molecule with the maximum fitness value until the $(t+1)$-th iteration is updated as 320 the global optimal solution $\bar{\rho}_{\text {best }}^{t+1}$. The iteration ends when the QCRO algorithm 321 achieves the terminal condition.

\subsection{Process of multiple-relay selection based on QCRO}

To tackle the MRS problem of (22) in secure massive MIMO two-way relay 324 networks, for QCRO algorithm, the fitness function is set as $f\left(\overline{\boldsymbol{x}}_{n}^{t}\right)=\left\{\begin{array}{ll}C_{\text {sum }}\left(\overline{\boldsymbol{x}}_{n}^{t}\right), & \text { satisfy constraint conditions } \\ 0, & \text { else }\end{array}\right.$ For each quantum molecule, the measurement state is corresponding to the MRS result which should be optimized.

327 Hence, the optimal MRS result corresponds to the global optimal solution of QCRO 

optimization can be shown in Algorithm 1.

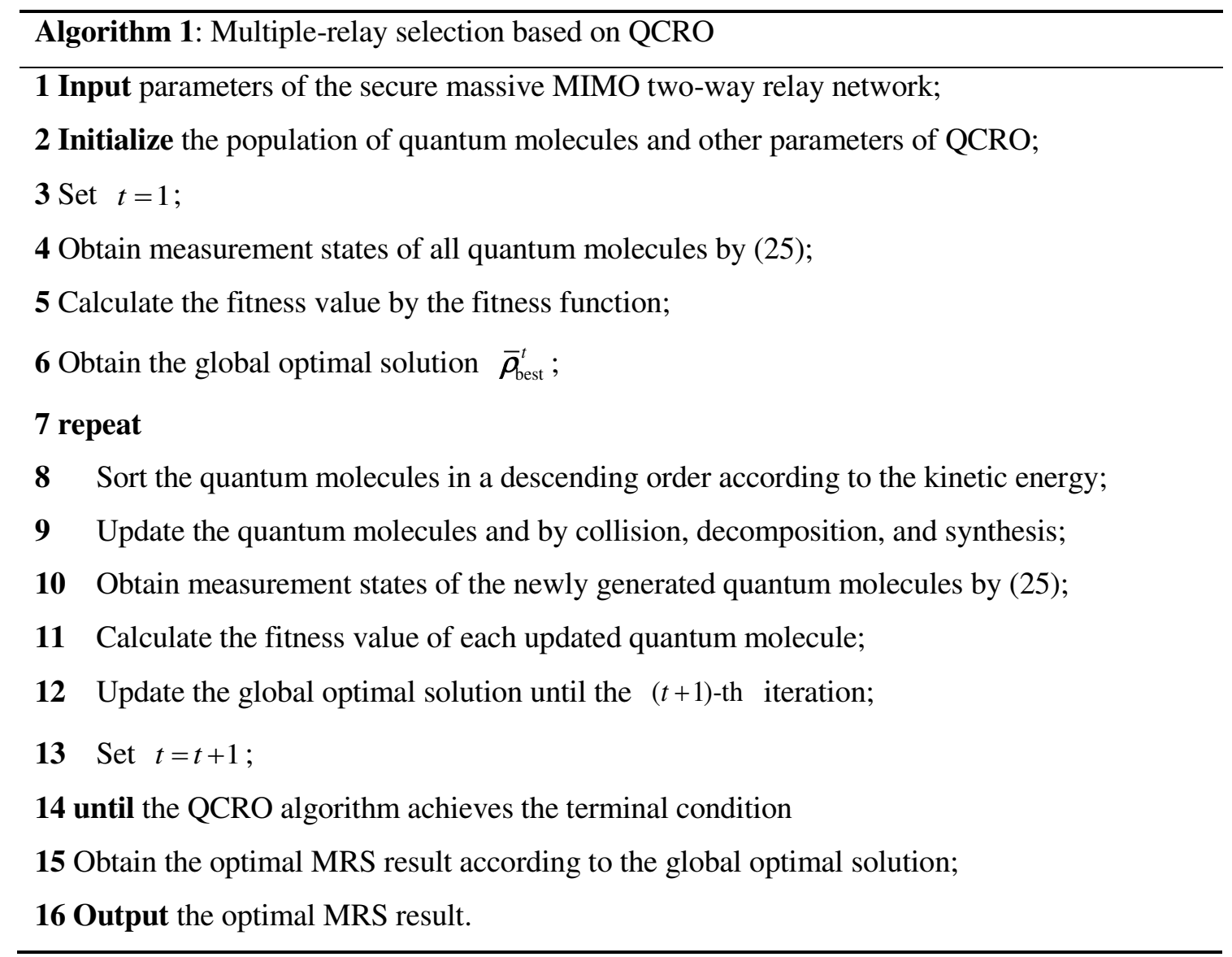

Here we present the secrecy performance in secure massive MIMO two-way relay networks. We consider a two-dimensional network topology where $L$ single-antenna relays are randomly located at $(50,0)$ with the radius of 20 , the $\mathrm{BS}$ and the legitimate user are located at the positions $(0,0)$ and $(100,0)$ (unit: meters), respectively. $K$ single-antenna eavesdroppers are randomly located in the system. We set $M_{t}=128$, $\xi=3.8, L=20$, and $I_{\text {th }}=-20 \mathrm{dBm}$. The system bandwidth $B=100 \mathrm{MHz}$, and noise power spectral density $N_{0}=-174 \mathrm{dBm} / \mathrm{Hz}$. To reduce the implementation complexity, maximum ratio transmission (MRT) and maximum ratio combining (MRC) methods are adopted at the BS for precoding and receiving. The comparisons of the proposed 
QCRO algorithm, existing intelligent algorithms and relay selection strategies are presented in the first part. For the second part, we illustrate the impact on the secrecy sum-rate of the cooperative transmission mode based on QCRO algorithm with various system parameters. All results are the average of 200 Monte-Carlo simulations.

\subsection{Performance comparisons with QCRO}

The comparisons of QCRO algorithm, particle swarm optimization (PSO) algorithm [40], chemical reaction optimization (CRO) algorithm [39], single-relay selection (SRS) strategy [32], and random multiple-relay selection (RMRS) strategy on the security performance are presented in Figs. 1 - 5. For comparison purpose, we set the maximum number of iterations for QCRO, PSO, and CRO algorithms to the same value, and all these algorithms are set to the same population size. The other parameters of PSO and CRO algorithms are set to the optimal values cited in [40] and [39], respectively. For QCRO algorithm, all quantum bits are initialized to 0.5 and the initial kinetic energy each quantum molecule is set to 1000. The parameter settings of QCRO algorithm are shown in Table 2.

Table 2 Parameter settings of QCRO algorithm

\begin{tabular}{cc}
\hline Parameter & Value \\
\hline Population size $(N)$ & 60 \\
Maximum iteration number & 500 \\
Loss rate of kinetic energy $(\Delta e)$ & 0.2 \\
Reaction ratio of collision $\left(\mu_{1}\right)$ & 0.55 \\
Reaction ratio of synthesis $\left(\mu_{2}\right)$ & 0.15 \\
Reaction ratio of decomposition $\left(\mu_{3}\right)$ & 0.3 \\
Weight coefficient $\left(c_{1}\right)$ & 0.1 \\
Weight coefficient $\left(c_{2}\right)$ & 0.1 \\
Weight coefficient $\left(c_{3}\right)$ & 0.03 \\
Weight coefficient $\left(c_{4}\right)$ & 0.03 \\
Weight coefficient $\left(c_{5}\right)$ & 0.03 \\
Mutation probability $\left(\kappa_{1}\right)$ & 0.5 \\
Conversion probability $\left(\kappa_{2}\right)$ & $0.1 / L$ \\
\hline
\end{tabular}


359 SRS, and RMRS strategies are presented in Fig. 1 with $p_{B S}=35 \mathrm{dBm}, p_{u}=30 \mathrm{dBm}$, $p_{\max }=30 \mathrm{dBm}$, and $K=1$. We observe that QCRO algorithm has a fast convergence

361 speed and a higher convergent accuracy throughout the iterations. For the MRS problem of (22), traditional CRO and PSO algorithms are prone to fall into local optimum in iteration processes. Since the proposed QCRO combines the merits of quantum calculation and preserves the diversity of chemical reaction process, QCRO has the strong optimization ability for solving complicated optimization problems. The results also illustrate that the prominent advantage of QCRO over the SRS and RMRS strategies on secrecy sum-rate in a massive MIMO system.

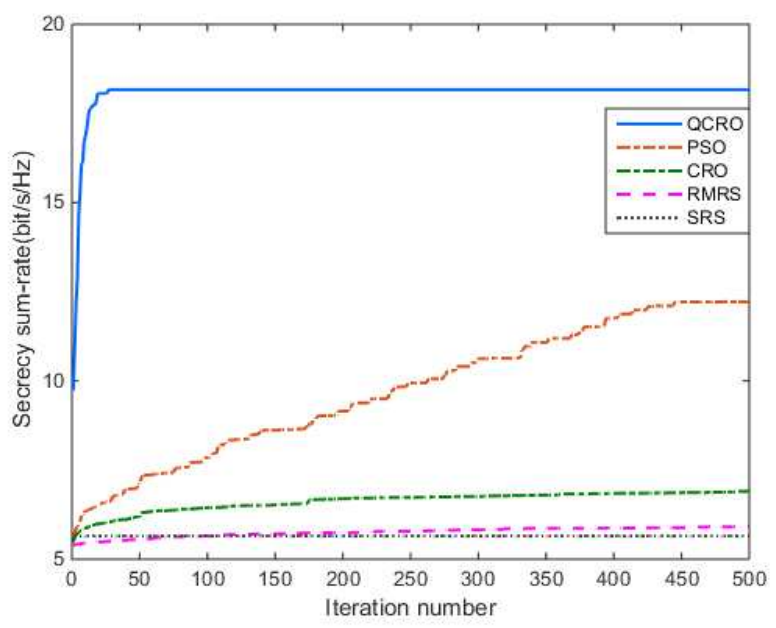

Fig. 1 Convergence performance comparisons

The security performance of QCRO, PSO, CRO, RMRS and SRS with the variation of $p_{B S}, p_{u}, p_{\max }$, and $K$ are shown in Figs. $2-5$. In Fig. 2, for most strategies, the secrecy sum-rate of the massive MIMO system increases along with $p_{B S}$. But the rising tendency begins to slow down when $p_{B S}$ is over $15 \mathrm{dBm}$. This phenomenon is caused by information leakage, which has a negative influence on the secrecy performance. According to (3) and (13), the eavesdropper may obtain more information from the BS with a higher $p_{B S}$, and the secrecy sum-rate may decrease 


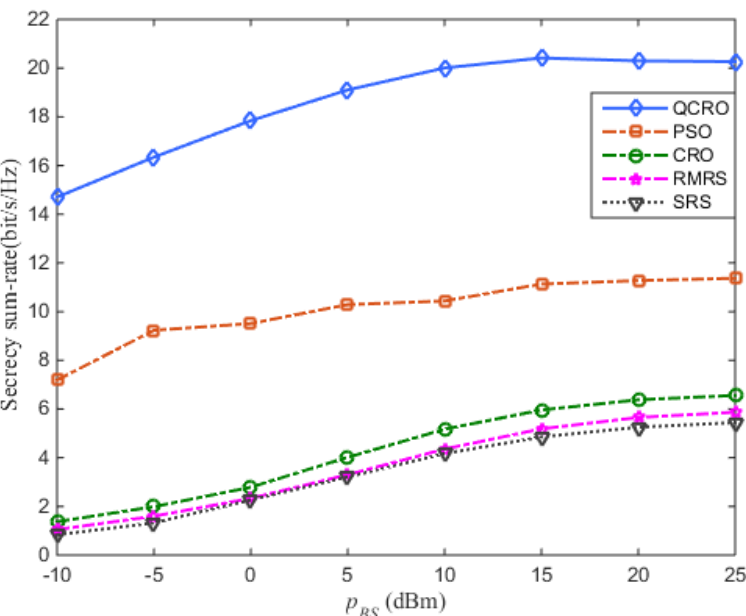

Fig. 2 Secrecy sum-rate in different $p_{B S}$ with $p_{u}=30 \mathrm{dBm}, \quad p_{\max }=30 \mathrm{dBm}$, and $K=1$

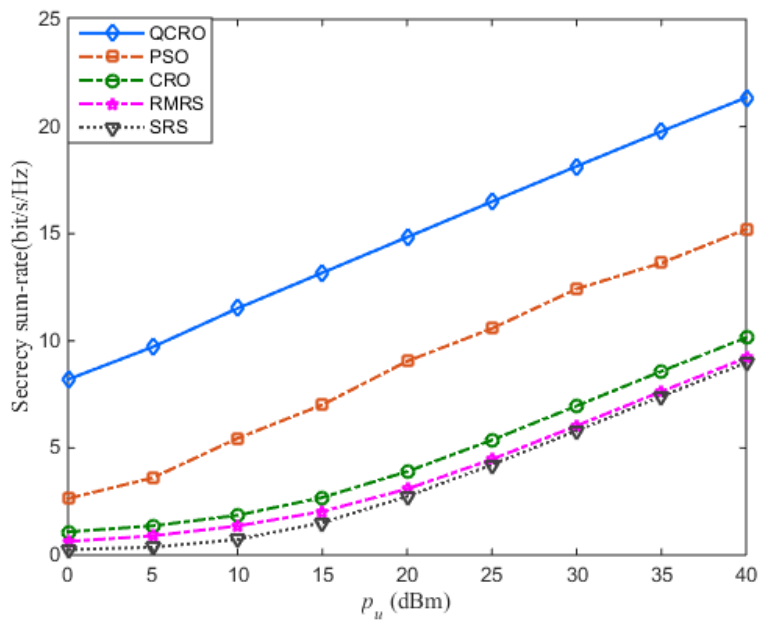

Fig. 3 Secrecy sum-rate in different $p_{u}$ with $p_{B S}=35 \mathrm{dBm}, \quad p_{\max }=30 \mathrm{dBm}$, and $K=1$

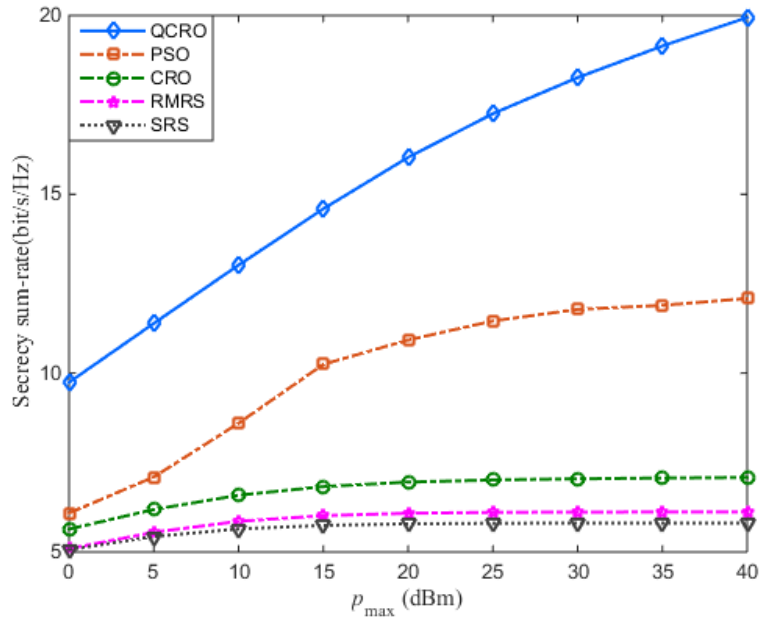

Fig. 4 Secrecy sum-rate in different $p_{\max }$ with $p_{B S}=35 \mathrm{dBm}, \quad p_{u}=30 \mathrm{dBm}$, and $K=1$ 

maximum transmit power of the cooperative relays on the security performance. For all strategies in Fig. 3, a higher secrecy sum-rate can be achieved by increasing $p_{u}$. The results in Fig. 4 also illustrate that increasing $p_{\max }$ can boost the security performance. The reason is that a larger $p_{\max }$ will permit relays to broadcast the signals at a higher transmission power under certain interference conditions. After self-interference elimination, the increment of SINR at the legitimate devices is higher than that of the eavesdroppers.

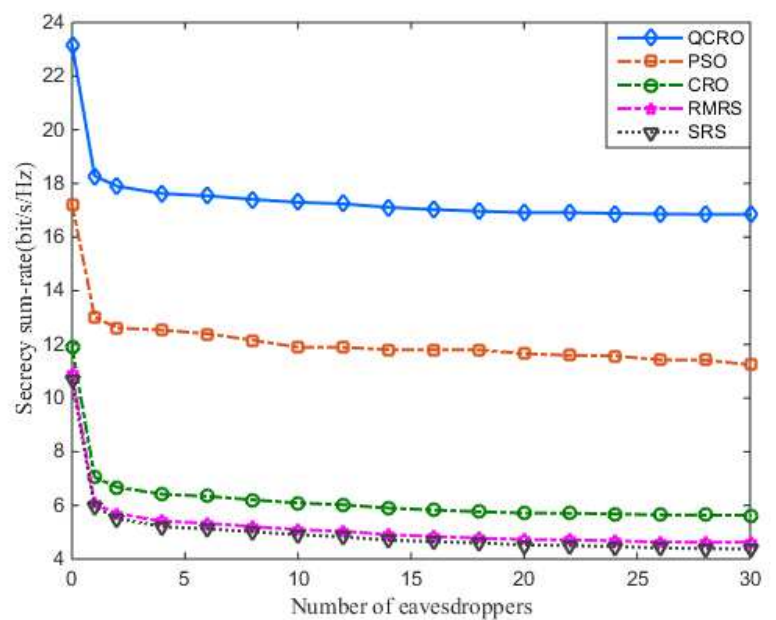

Fig. 5 Secrecy sum-rate in different $K$ with $p_{B S}=35 \mathrm{dBm}, p_{u}=30 \mathrm{dBm}$, and $p_{\max }=30 \mathrm{dBm}$

The impact of different number of eavesdroppers on the secrecy sum-rate is presented in Fig. 5. The result shows that the existence of eavesdroppers has an adverse effect on the secrecy performance, and the information leakage increases along with $K$. That is because by increasing $K$, the probability of emerging an eavesdropper with a higher channel gain increases accordingly. For the non-colluding eavesdroppers, the information leakage is determined by the maximum received SINR at the eavesdroppers during the two transmission phases. From Figs. 2 - Fig. 5, we conclude that QCRO has great advantages over other strategies in improving the communication security of secure massive MIMO two-way relay networks. 


\subsection{Impact of different system parameters}

The performance of the proposed cooperative transmission mode based on QCRO algorithm in different $p_{u}$ and $p_{B S}$ is studied in Fig. 6, where $p_{u}$ increases from $0 \mathrm{dBm}$ to $40 \mathrm{dBm}, p_{B S}=0 \mathrm{dBm}, 3 \mathrm{dBm}, 5 \mathrm{dBm}$, and $10 \mathrm{dBm}$, respectively. From the simulations, the system can obtain a higher secrecy sum-rate with a larger level of $p_{u}$ at first. But when $p_{u}$ is over $30 \mathrm{dBm}$, the increment of secrecy sum-rate begins to slow down. For the cases of $p_{B S}=3 \mathrm{dBm}, 5 \mathrm{dBm}$, and $10 \mathrm{dBm}$, the secrecy sum-rate decreases with $p_{u}$ when $p_{u}$ is over $35 \mathrm{dBm}$. The reason is that the SINR received at the eavesdropper increases with $p_{u}$, which may cause more information leakage. Hence, we can appropriately increase $p_{B S}$ to confuse the eavesdroppers.

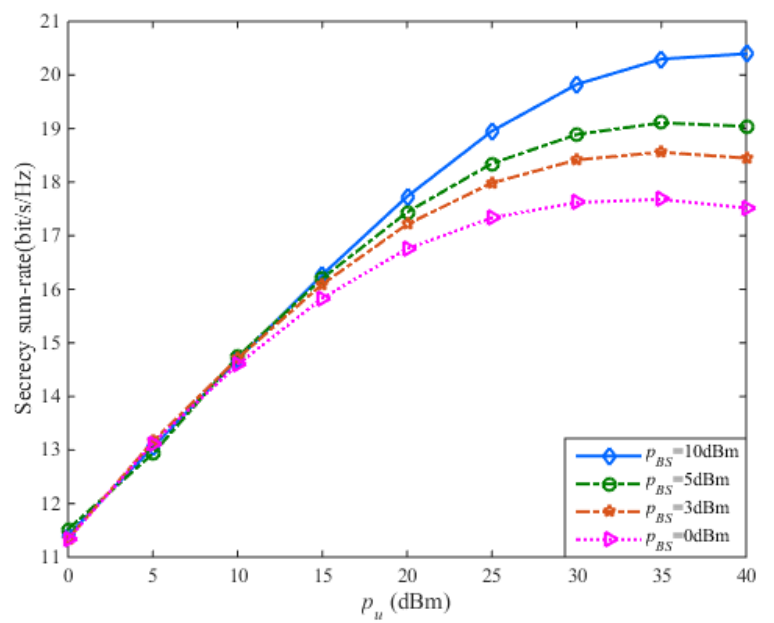

Fig. 6 Secrecy sum-rate in different $p_{u}$ and $p_{B S}$ with $p_{\max }=30 \mathrm{dBm}$ and $K=1$

In Fig. 7, we investigate the impact of different $p_{\max }$ and $p_{u}$ levels with $p_{\max }$ varying from $0 \mathrm{dBm}$ to $40 \mathrm{dBm}, p_{B S}=0 \mathrm{dBm}, 5 \mathrm{dBm}, 10 \mathrm{dBm}, 20 \mathrm{dBm}$, and $30 \mathrm{dBm}$, respectively. From the simulations, the increasing trend of secrecy sum-rate with $p_{u}$ is in accordance with the results of Fig. 3. We also observe that both higher levels of $p_{\max }$ and $p_{u}$ can help boost the communication security. The reason is that a higher $p_{\max }$ can permit each relay to use more power for its own transmission. With the 
422 of the eavesdroppers according to the SIC criterion.

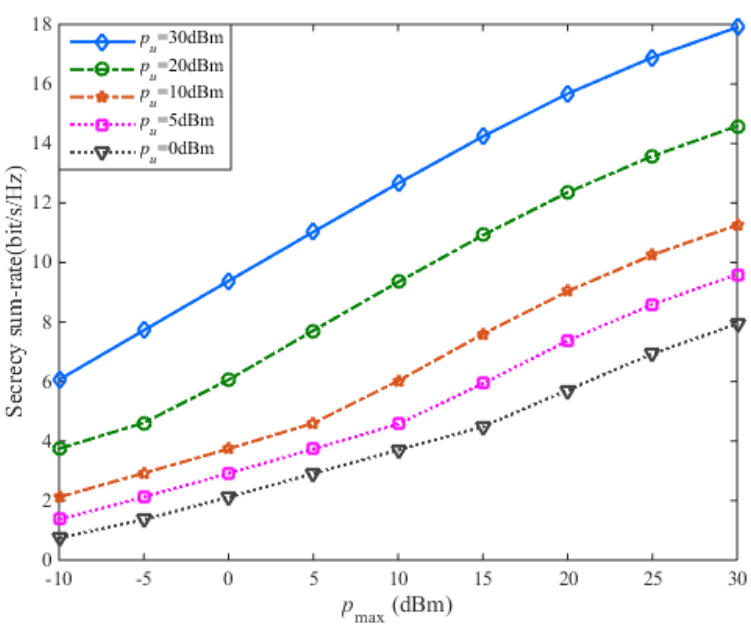

Fig. 7 Secrecy sum-rate in different $p_{\max }$ and $p_{u}$ with $p_{B S}=35 \mathrm{dBm}$ and $K=1$

The influence of $p_{B S}$ and number of eavesdroppers are studied in Fig. 8. In simulations, $p_{B S}$ varying from $-10 \mathrm{dBm}$ to $20 \mathrm{dBm}$, and $K=1,3,10,20$, respectively. The results indicate that a larger number of eavesdroppers can cause more information leakage, which jeopardizes the communication security of massive MIMO system. For a certain value of $K$, we can obtain a higher secrecy sum-rate with a larger $p_{B S}$.

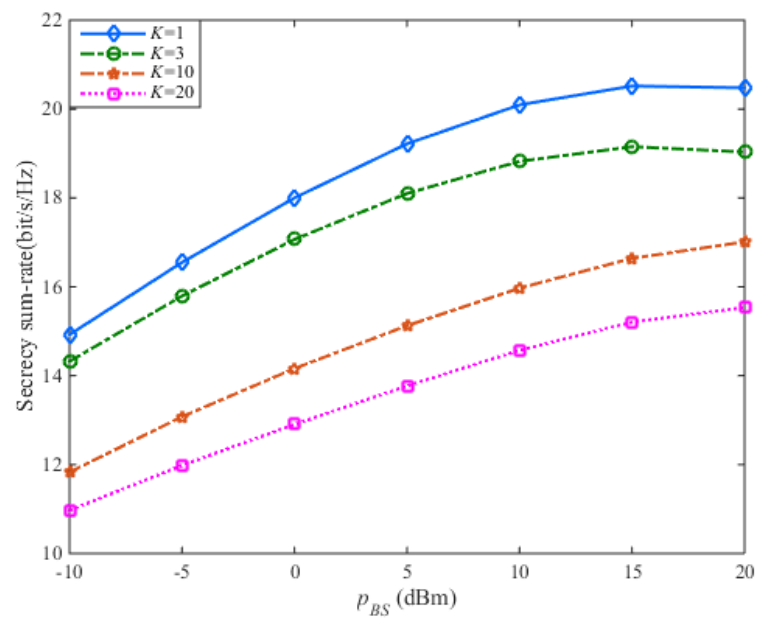

Fig. 8 Secrecy sum-rate in different $p_{B S}$ and $K$ with $p_{u}=30 \mathrm{dBm}$ and $p_{\max }=30 \mathrm{dBm}$ The influence of different $p_{\max }$ and $L$ in single and multiple eavesdropper cases are investigated in Fig. 9. We consider $K=1$ and $K=10, p_{\max }$ varies from $-10 \mathrm{dBm}$ 
to $20 \mathrm{dBm}$, and $L$ increases from 5 to 20 . The simulation results indicate that both

higher $p_{\max }$ and more relays can boost the transmission security. Obviously, the secrecy sum-rate in the case of single eavesdropper is higher than that of multiple eavesdroppers.

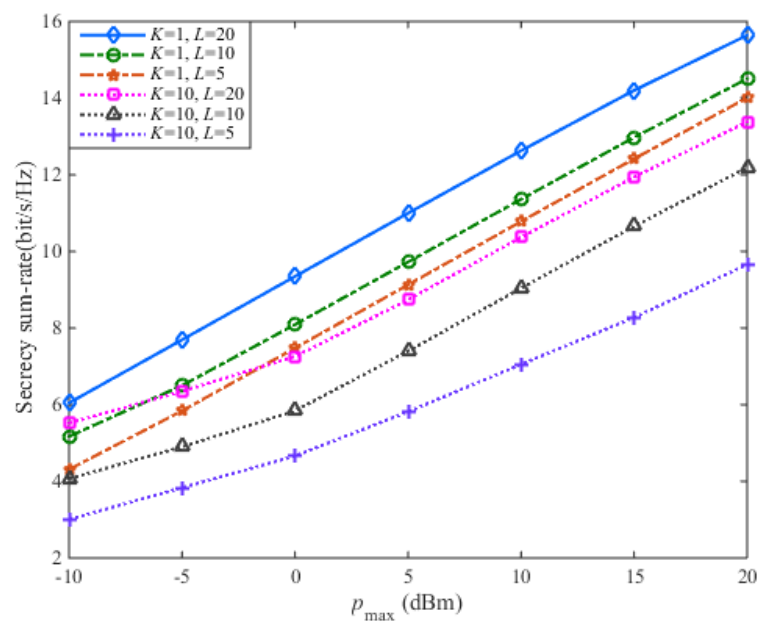

Fig. 9 Secrecy sum-rate in different $p_{\max }$ and $L$ with $p_{B S}=35 \mathrm{dBm}, p_{u}=30 \mathrm{dBm}$, and

$$
K=\{1,10\}
$$

\section{Conclusions}

In this paper, we have designed a cooperative transmission mode to achieve the trade-off between the communication security and reliability in a massive MIMO system with two-way relay cooperation. Based on the multiple-relay collaboration strategy, some relays function as helper while other relays act as jammer to against the interception of eavesdroppers. Considering the system capacity, energy conservation, and QoS requirements, we have formulated the MRS problem in a secure massive MIMO two-way relay network. Then, we have introduced the implementation of multiple-relay collaboration strategy based on QCRO to optimize the secrecy sum-rate and simulation results have demonstrated its effectiveness in different communication scenarios. In future research, we will incorporate the efforts of this work with energy harvesting, network slicing, and ultra-dense heterogeneous 
networks to meet the increasing demands of future wireless communication systems.

\section{Abbreviations}

IoE: Internet of Everything; MIMO: Multiple-input multiple-output; BS: Base station; QCRO: Quantum chemical reaction optimization; QoS: Quality of service; DT: Direct transmission; OWR: One-way relaying; TWR: Two-way relaying; MRS: Multiple-relay selection; CSI: Channel state information; TDD: Time-division duplexed; CRCJ: Cooperative relaying and cooperative jamming; AWGN: Additive white Gaussian noise; SINR: Signal-to-interference plus noise ratio; SIC: Self-interference cancellation; CRO: Chemical reaction optimization; KE: Kinetic energy; MRT: Maximum ratio transmission; MRC: Maximum ratio combining; PSO: Particle swarm optimization; SRS: Single-relay selection; RMRS: Random multiple-relay selection.

\section{Acknowledgments}

The authors would like to acknowledge the anonymous reviewers and editors for their efforts in valuable comments and suggestions

\section{Authors' contributions}

Yumeng Su carried out experiments and theoretical analysis, and was a major contributor in writing the manuscript. Hongyuan Gao mainly provided the guidance for deriving these expressions. Shibo Zhang conceived of the study, and participated in its design and coordination. All authors read and approved the final manuscript.

\section{Funding}

This work was supported by the National Natural Science Foundation of China (No. 61571149), the Special China Postdoctoral Science Foundation (2015T80325), Fundamental Research Funds for the Central Universities (HEUCFP201808 and HEUCF190801), and the China Postdoctoral Science Foundation (2013M530148).

\section{Availability of data and materials}

All data generated or analysed during this study are included in this article. 


\section{Competing interests}

479 The authors declare that they have no competing interests.

480

481

482

483

484

485

486

487

488

489

490

491

492

493

494

495

496

497

498

499

500

501

502

503

504

505

506

\section{References}

1. P. Ramezani, A. Jamalipour, Toward the evolution of wireless powered communication networks for the future Internet of Things. IEEE Netw. 31(6), 62-69 (2017).

2. S. J. Nawaz, S. K. Sharma, S. Wyne, et al., Quantum machine learning for $6 \mathrm{G}$ communication networks: state-of-the-art and vision for the future. IEEE Access 7, 46317-46350 (2019).

3. T. S. Rappaport, Y. C. Xing, O. Kanhere, et al., Wireless communications and applications above 100 GHZ: opportunities and challenges for 6G and beyond. IEEE Access 7, 78729-78757 (2019).

4. P. Yang, Y. Xiao, M. Xiao, et al., 6G wireless communications: Vision and potential techniques. IEEE Netw. 33(4), 70-75 (2019).

5. L. Zhang, Y. C. Liang, D. Niyato, 6G visions: mobile ultra-broadband, super Internet-of-Things, and artificial intelligence. China Commun. 16(8), 1-14 (2019).

6. T. J. Lv, Z. P. Lin, P. M. Huang, et al., Optimization of the energy-efficient relay-based massive IoT network. IEEE Internet Things J. 5(4), 3043-3058 (2018).

7. D. B. Rawat, T. White, M. S. Parwez, et al., Evaluating secrecy outage of physical layer security in large-scale MIMO wireless communications for cyber-physical systems. IEEE Internet Things J. 4(6), 1987-1993 (2017).

8. E. G. Larsson, O. Edfors, F. Tufvesson, et al., Massive MIMO for next generation wireless systems. IEEE Commun. Mag. 52(2), 186-195 (2014).

9. H. Y. Gao, Y. M. Su, S. B. Zhang, et al., Antenna selection and power allocation design for 5G massive MIMO uplink networks. China Commun. 16(4), 1-15 (2019).

10. A. Q. He, L. F. Wang, M. Elkashlan, et al., Spectrum and energy efficiency in massive MIMO enabled Hetnets: a stochastic geometry approach. IEEE Commun. Lett. 19(12), 2294-2297 (2015).

11. L. X. Li, A. P. Petropulu, Z. Chen, MIMO secret communications against an active eavesdropper. IEEE Trans. Inf. Forensic Secur. 12(10), 2387-2401 (2017).

12. K. W. Jiang, T. Jing, Y. Huo, et al., SIC-based secrecy performance in uplink NOMA multi-eavesdropper wiretap channels. IEEE Access 6, 19664-19680 (2018). 
13. S. Y. Liu, Y. Hong, E. Viterbo, Unshared secret key cryptography. IEEE Trans. Wirel. Commun. 13(12), 6670-6683 (2014).

14. S. X. Wang, W. Li, J. Lei, Physical-layer encryption in massive MIMO systems with spatial modulation. China Commun. 15(10), 159-171 (2018).

15. I. Grigg, P. Gutmann, The curse of cryptographic numerology. IEEE Secur. Priv. 9(3), 70-72 (2011).

16. R. Zi, J. Liu, L. Gu, et al., Enabling security and high energy efficiency in the Internet of Things with massive MIMO hybrid precoding. IEEE Internet Things J. 6(5), 8615-8625 (2019).

17. Y. O. Basciftci, C. E. Koksal, A. Ashikhmin, Physical-layer security in TDD massive MIMO. IEEE Trans. Inf. Theory 64(11), 7359-7380 (2018).

18. F. C. Zhu, F. F. Gao, H. Lin, et al., Robust beamforming for physical layer security in BDMA massive MIMO. IEEE J. Sel. Areas Commun. 36(4), 775-787 (2018).

19. Y. P. Wu, R. Schober, D. W. K. Ng, et al., Secure massive MIMO transmission with an active eavesdropper. IEEE Trans. Inf. Theory 62(7), 3880-3900 (2016).

20. J. Zhu, R. Schober, V. K. Bhargava, Linear precoding of data and artificial noise in secure massive MIMO systems. IEEE Trans. Wirel. Commun. 15(3), 2245-2261 (2016).

21. A. Kuhestani, A. Mohammadi, M. Mohammadi, Joint relay selection and power allocation in large-scale MIMO systems with untrusted relays and passive eavesdroppers. IEEE Trans. Inf. Forensic Secur. 13(2), 341-355 (2018).

22. Y. L. Zou, X. B. Wang, W. M. Shen, et al., Security versus reliability analysis of opportunistic relaying. IEEE Trans. Veh. Technol. 63(6), 2653-2661 (2014).

23. C. Wang, H. M. Wang, X. G. Xia, Hybrid opportunistic relaying and jamming with power allocation for secure cooperative networks. IEEE Trans. Wirel. Commun. 14(2), 589-605 (2015).

24. J. Chen, X. M. Chen, T. Liu, et al., Toward green and secure communications over massive MIMO relay networks: joint source and relay power allocation. IEEE Access 5, 869-880 (2017).

25. D. Kudathanthirige, S. Timilsina, G. A. A. Baduge, Secure communication in relay-assisted massive MIMO downlink with active pilot attacks. IEEE Trans. Inf. Forensic Secur. 14(11), 2819-2833 (2019).

26. T. M. Hoang, T. Q. Duong, H. D. Tuan, et al., Secure massive MIMO relaying systems in a Poisson field of eavesdroppers. IEEE Trans. Commun. 65(11), 4857-4870 (2017). 
27. X. M. Chen, J. Chen, T. Liu, Secure transmission in wireless powered massive MIMO relaying systems: performance analysis and optimization. IEEE Trans. Veh. Technol. 65(10), 8025-8035 (2016).

28. T. Mekkawy, R. G. Yao, N. Qi, et al., Secure relay selection for two way amplify-and-forward untrusted relaying networks. IEEE Trans. Veh. Technol. 67(12), 11979-11987 (2018).

29. D. Wang, B. Bai, W. Chen, et al., Secure green communication via untrusted two-way relaying: a physical layer approach. IEEE Trans. Commun. 64(5), 1861-1874 (2016).

30. K. Lee, J. P. Hong, H. H. Choi, et al., Wireless-powered two-way relaying protocols for optimizing physical layer security. IEEE Trans. Inf. Forensic Secur. 14(1), 162-174 (2019).

31. J. C. Chen, R. Q. Zhang, L. Y. Song, et al., Joint relay and jammer selection for secure two-way relay networks. IEEE Trans. Inf. Forensic Secur. 7(1), 310-320 (2012).

32. C. S. Zhang, J. H. Ge, F. K. Gong, et al., Improving physical-layer security for wireless communication systems using duality-aware two-way relay cooperation. IEEE Syst. J. 13(2), 1241-1249 (2019).

33. C. S. Zhang, J. H. Ge, J. Li, F. K. Gong, et al., Complexity-aware relay selection for 5G large-scale secure two-way relay systems. IEEE Trans. Veh. Technol. 66(6), 5462-5466 (2017).

34. B. Dutta, R. Budhiraja, R. D. Koilpillai, et al., Analysis of quantized MRC-MRT precoder for FDD massive MIMO two-way AF relaying. IEEE Trans. Commun. 67(2), 988-1003 (2019).

35. J. J. Feng, S. D. Ma, G. H. Yang, et al., Power scaling of full-duplex two-way massive MIMO relay systems with correlated antennas and MRC/MRT processing. IEEE Trans. Wirel. Commun. 16(7), 4738-4753 (2017).

36. J. Y. Zhang, X. P. Xue, E. Bjornson, et al., Spectral efficiency of multipair massive MIMO two-way relaying with hardware impairments. IEEE Wirel. Commun. Lett. 7(1), 14-17 (2018).

37. X. Pan, L. Guo, C. Dong, et al., in Proc. IEEE International Conference on Communications (ICC), Secure multi-pair massive MIMO two-way amplify-and-forward relay network with power allocation scheme (IEEE, Paris, 2017), pp. 1-5.

38. R. Hamdi, E. Driouch, W. Ajib, Energy management in hybrid energy large-scale MIMO systems. IEEE Trans. Veh. Technol. 66(11), 10183-10193 (2017).

39. A. Y. S. Lam, V. O. K. Li, J. J. Q. Yu, Power-controlled cognitive radio spectrum allocation with chemical reaction optimization. IEEE Trans. Wirel. Commun. 12(7), 3180-3190 (2013). 
40. S. K. Chou, M. K. Jiau, S. C. Huang, Stochastic set-based particle swarm optimization based on local exploration for solving the carpool service problem. IEEE Trans. Cybern. 46(8), 1771-1783 (2016).

\section{Figure title and legend}

Fig. 1 Convergence performance comparisons.

Blue line: QCRO; yellow line: PSO; green line: CRO; pink line: RMRS; black line: SRS.

Fig. 2 Secrecy sum-rate in different $p_{B S}$ with $p_{u}=30 \mathrm{dBm}, \quad p_{\max }=30 \mathrm{dBm}$, and $K=1$.

Blue line: QCRO; yellow line: PSO; green line: CRO; pink line: RMRS; black line: SRS.

Fig. 3 Secrecy sum-rate in different $p_{u}$ with $p_{B S}=35 \mathrm{dBm}, p_{\max }=30 \mathrm{dBm}$, and $K=1$.

Blue line: QCRO; yellow line: PSO; green line: CRO; pink line: RMRS; black line: SRS.

Fig. 4 Secrecy sum-rate in different $p_{\max }$ with $p_{B S}=35 \mathrm{dBm}, p_{u}=30 \mathrm{dBm}$, and $K=1$.

Blue line: QCRO; yellow line: PSO; green line: CRO; pink line: RMRS; black line: SRS.

Fig. 5 Secrecy sum-rate in different $K$ with $p_{B S}=35 \mathrm{dBm}, p_{u}=30 \mathrm{dBm}$, and $p_{\text {max }}=30 \mathrm{dBm}$ Blue line: QCRO; yellow line: PSO; green line: CRO; pink line: RMRS; black line: SRS.

Fig. 6 Secrecy sum-rate in different $p_{u}$ and $p_{B S}$ with $p_{\max }=30 \mathrm{dBm}$ and $K=1$.

Blue line: $p_{B S}=10 \mathrm{dBm}$; green line: $p_{B S}=5 \mathrm{dBm}$; yellow line: $p_{B S}=3 \mathrm{dBm}$; pink line: $p_{B S}=0 \mathrm{dBm}$

Fig. 7 Secrecy sum-rate in different $p_{\max }$ and $p_{u}$ with $p_{B S}=35 \mathrm{dBm}$ and $K=1$.

Blue line: $p_{u}=30 \mathrm{dBm}$; green line: $p_{u}=20 \mathrm{dBm}$; yellow line: $p_{u}=10 \mathrm{dBm}$; pink line: $p_{u}=5 \mathrm{dBm} ;$ black line: $p_{u}=0 \mathrm{dBm}$. 
594 Fig. 8 Secrecy sum-rate in different $p_{B S}$ and $K$ with $p_{u}=30 \mathrm{dBm}$ and $p_{\max }=30 \mathrm{dBm}$.

Blue line: $K=1$; green line: $K=3$; yellow line: $K=10$; pink line: $K=20$.

596

597

Fig. 9 Secrecy sum-rate in different $p_{\max }$ and $L$ with $p_{B S}=35 \mathrm{dBm}, \quad p_{u}=30 \mathrm{dBm}$, and 598 $K=\{1,10\}$.

599

Blue line: $K=1, L=20$; green line: $K=1, L=10$; yellow line: $K=1, L=5$; pink line:

600

$K=10, L=20$; black line: $K=10, L=10$; purple line: $K=10, L=5$.

601 
Figures

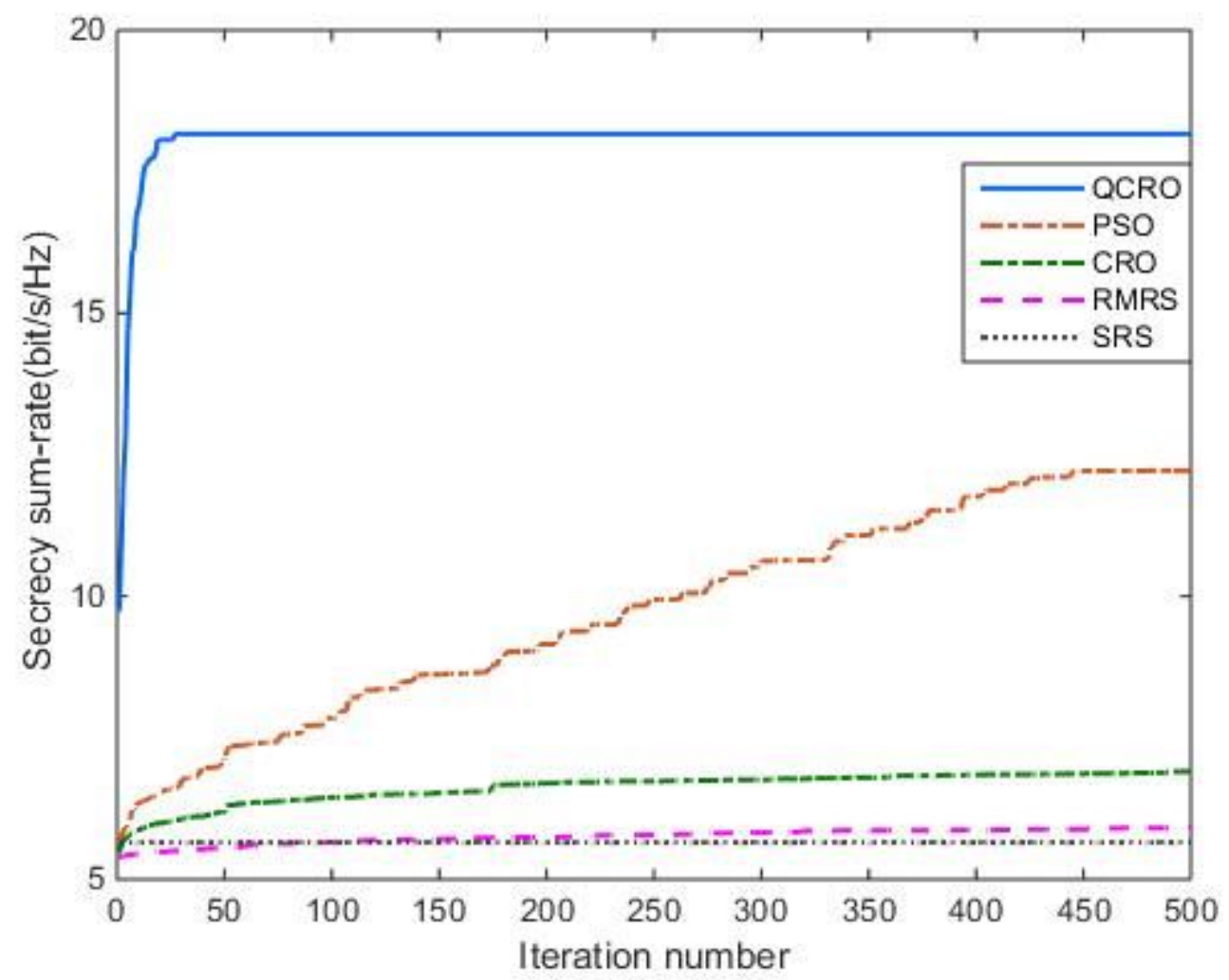

Figure 1

Convergence performance comparisons 


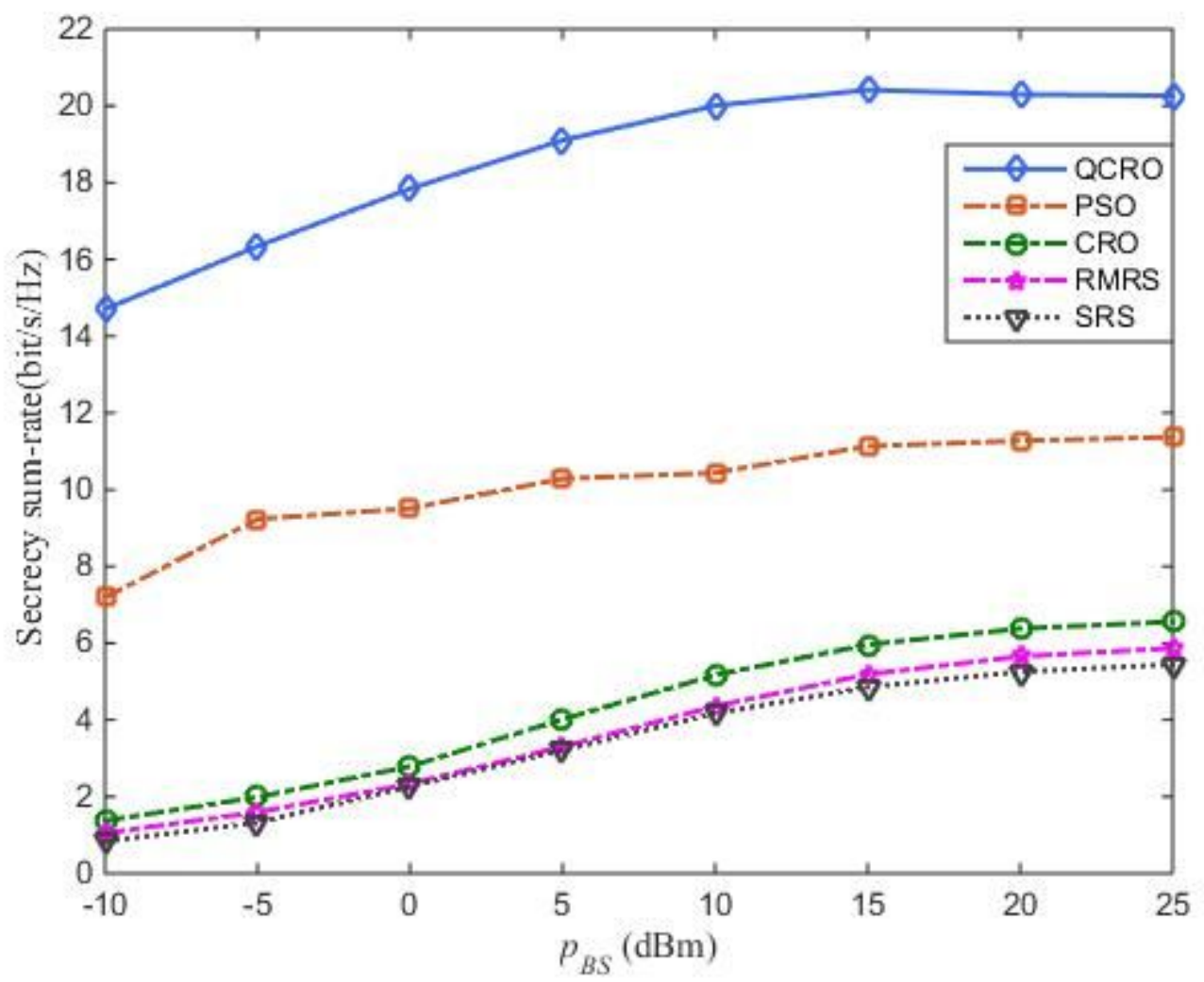

Figure 2

Secrecy sum-rate in different PBS with $\mathrm{Pu}=30 \mathrm{dBm}, \mathrm{Pmax}=30 \mathrm{dBm}$, and $\mathrm{K}=1$

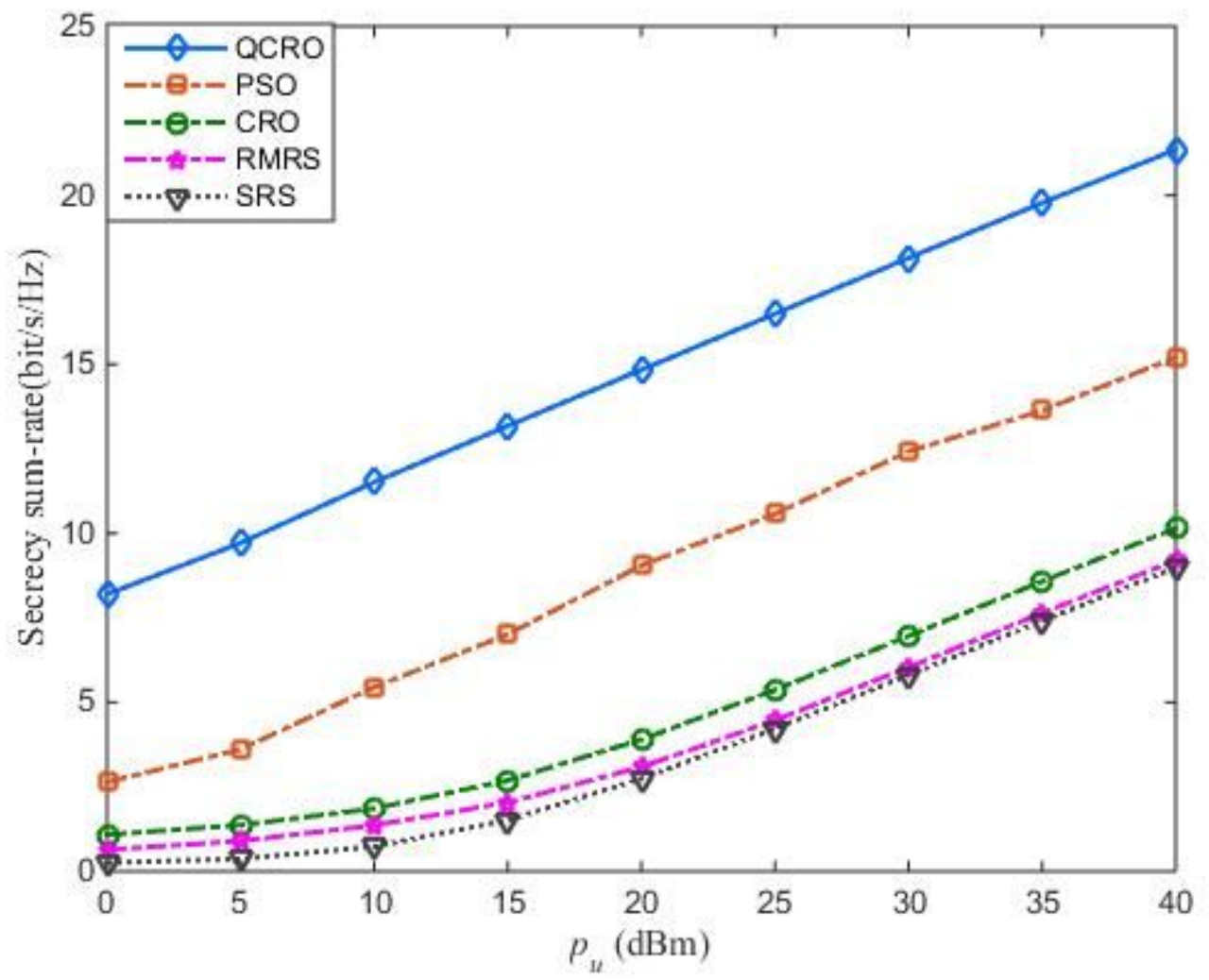


Figure 3

Secrecy sum-rate in different Pu with PBS $=35 \mathrm{dBm}, \mathrm{Pmax}=35 \mathrm{dBm}$, and $\mathrm{K}=1$

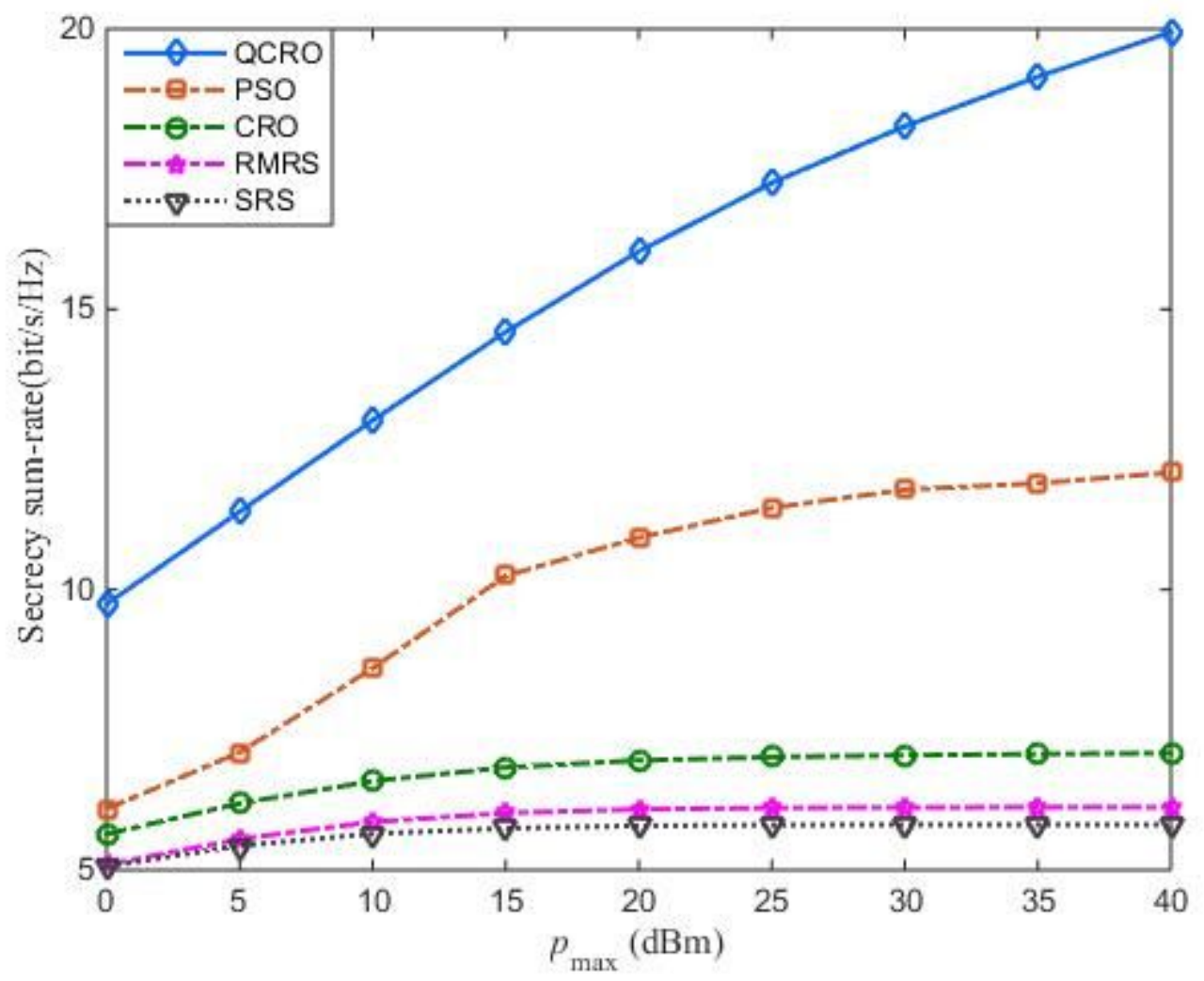

Figure 4

Secrecy sum-rate in different Pmax with PBS $=35 \mathrm{dBm}, \mathrm{Pu}=30 \mathrm{dBm}$, and $\mathrm{K}=1$ 


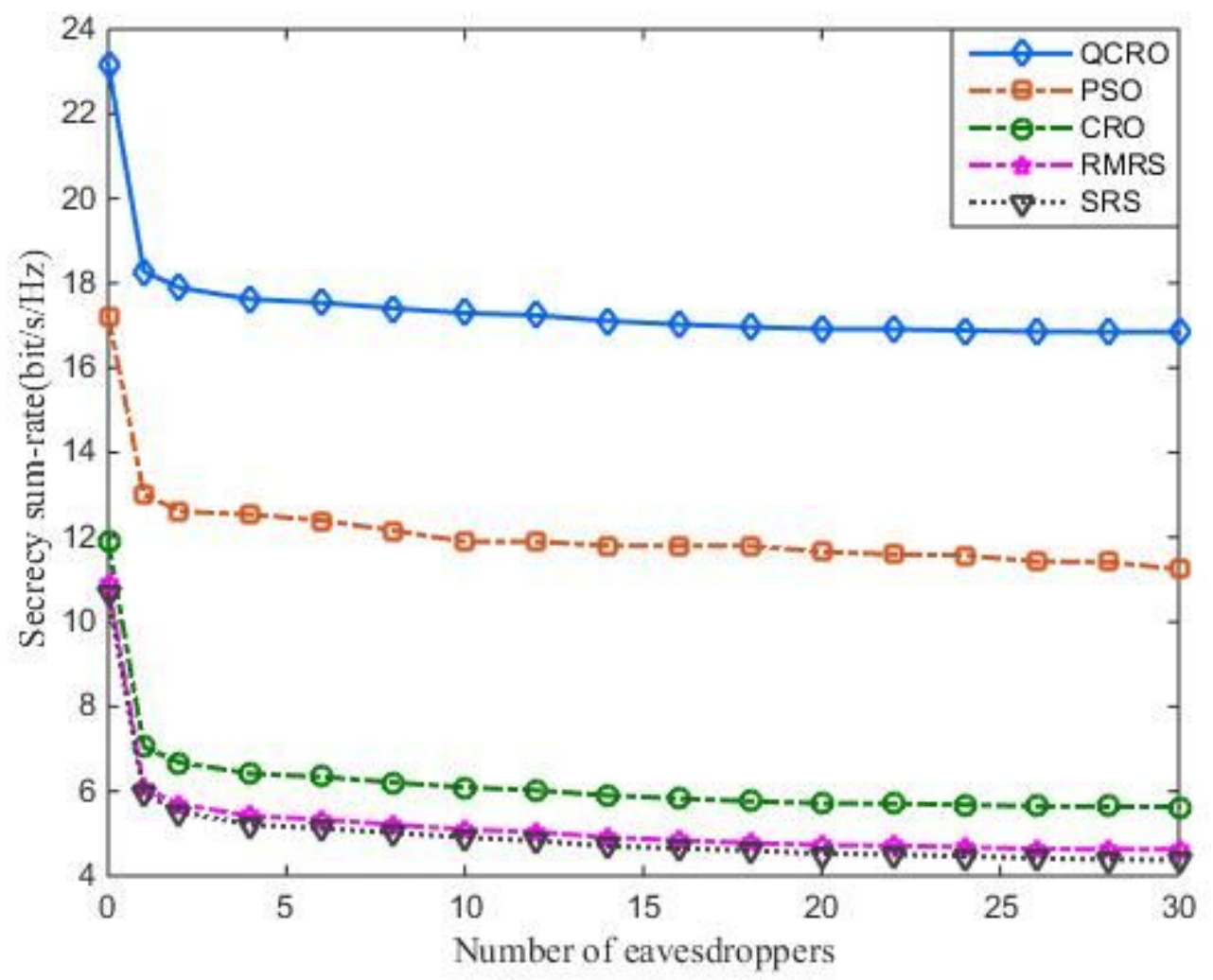

Figure 5

Secrecy sum-rate in different $\mathrm{K}$ with $\mathrm{PBS}=35 \mathrm{dBm}, \mathrm{Pu}=30 \mathrm{dBm}$, and $\mathrm{Pmax}=30 \mathrm{dBm}$

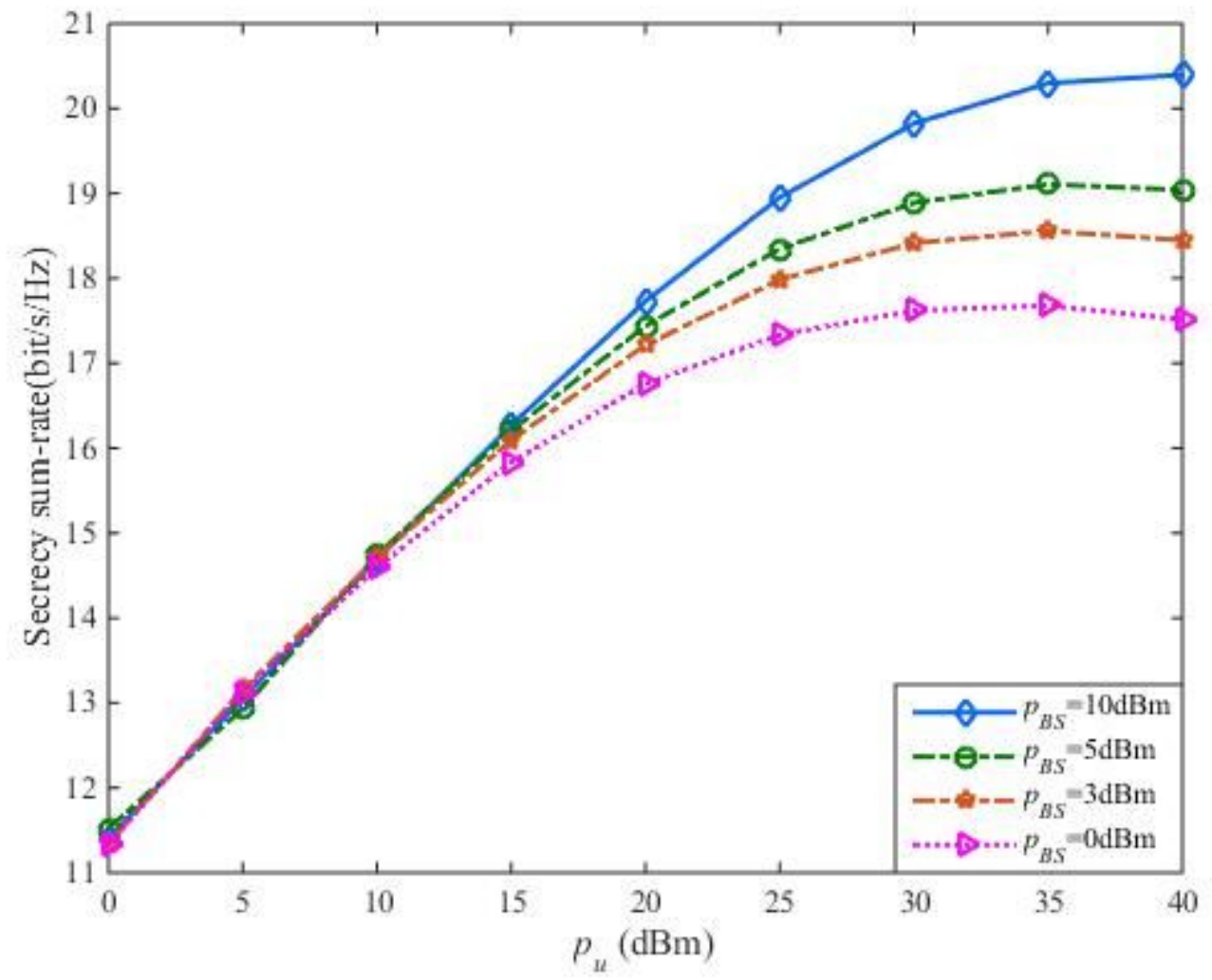


Figure 6

Secrecy sum-rate in different Pu with PBS with Pmax $=30 \mathrm{dBm}$, and $\mathrm{K}=1$

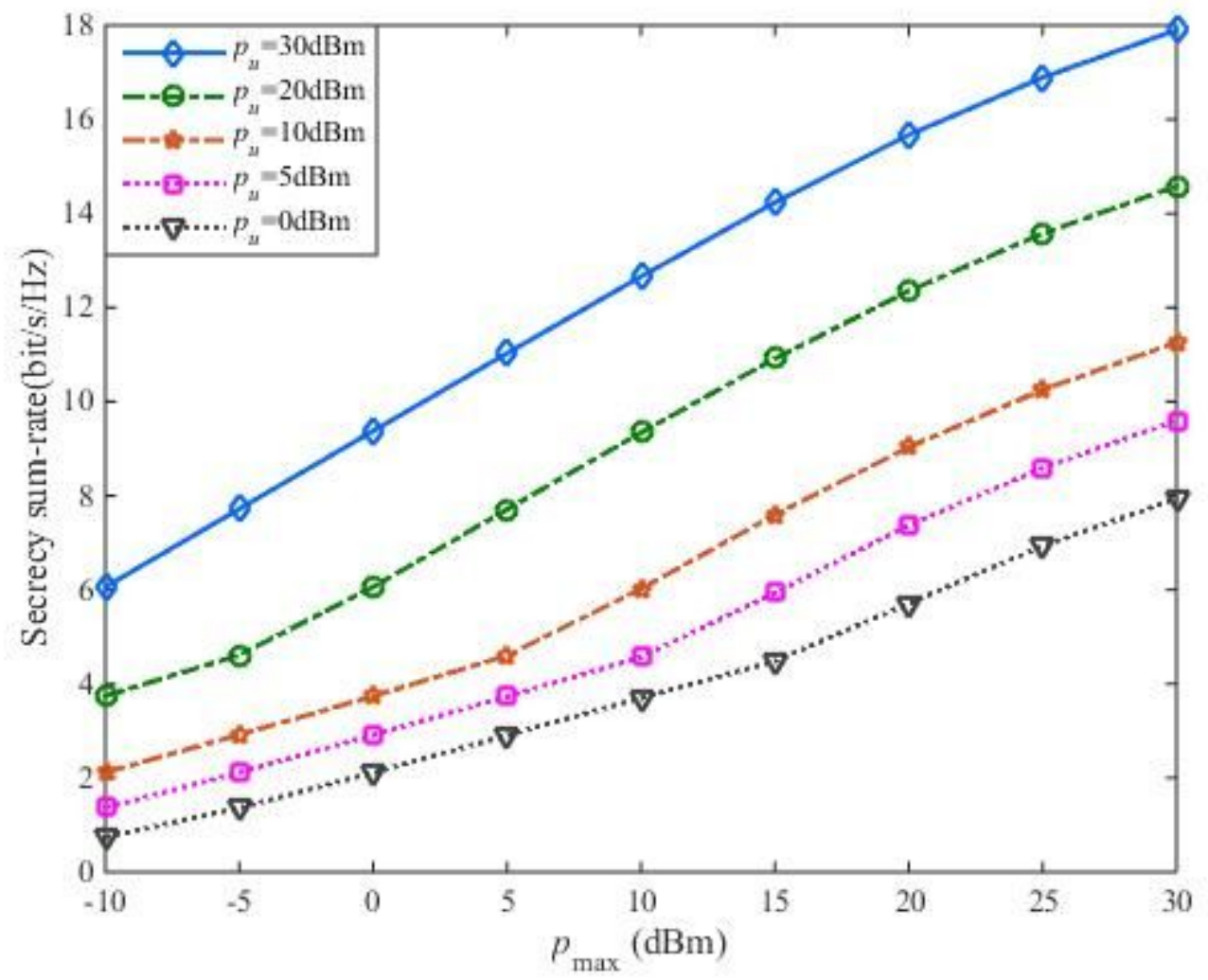

Figure 7

Secrecy sum-rate in different Pmax and Pu with PBS $=35 \mathrm{dBm}$, and $\mathrm{K}=1$ 


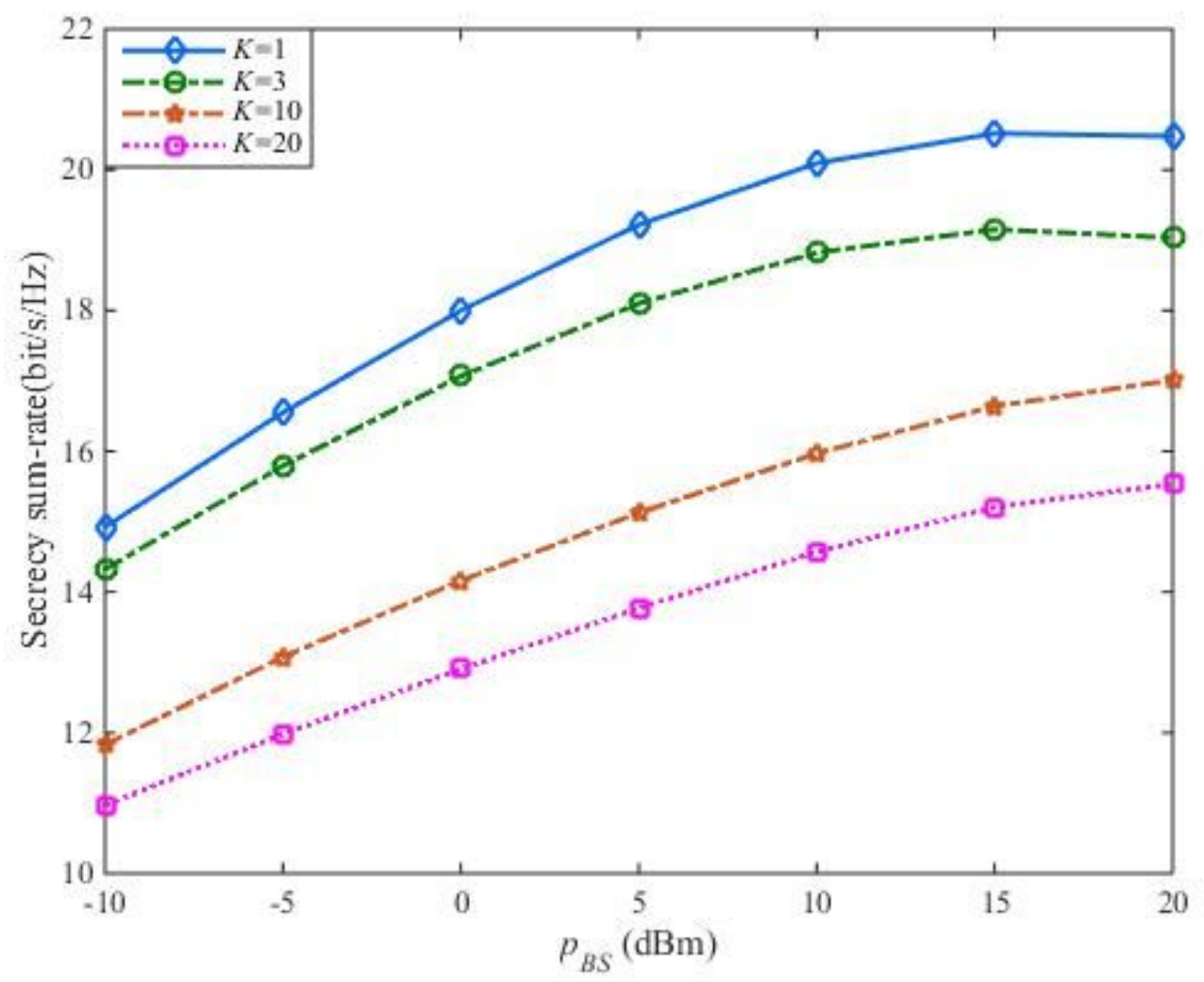

Figure 8

Secrecy sum-rate in different PBS and $\mathrm{K}$ with $\mathrm{Pu}=30 \mathrm{dBm}$, and pmax $=30 \mathrm{dBm}$

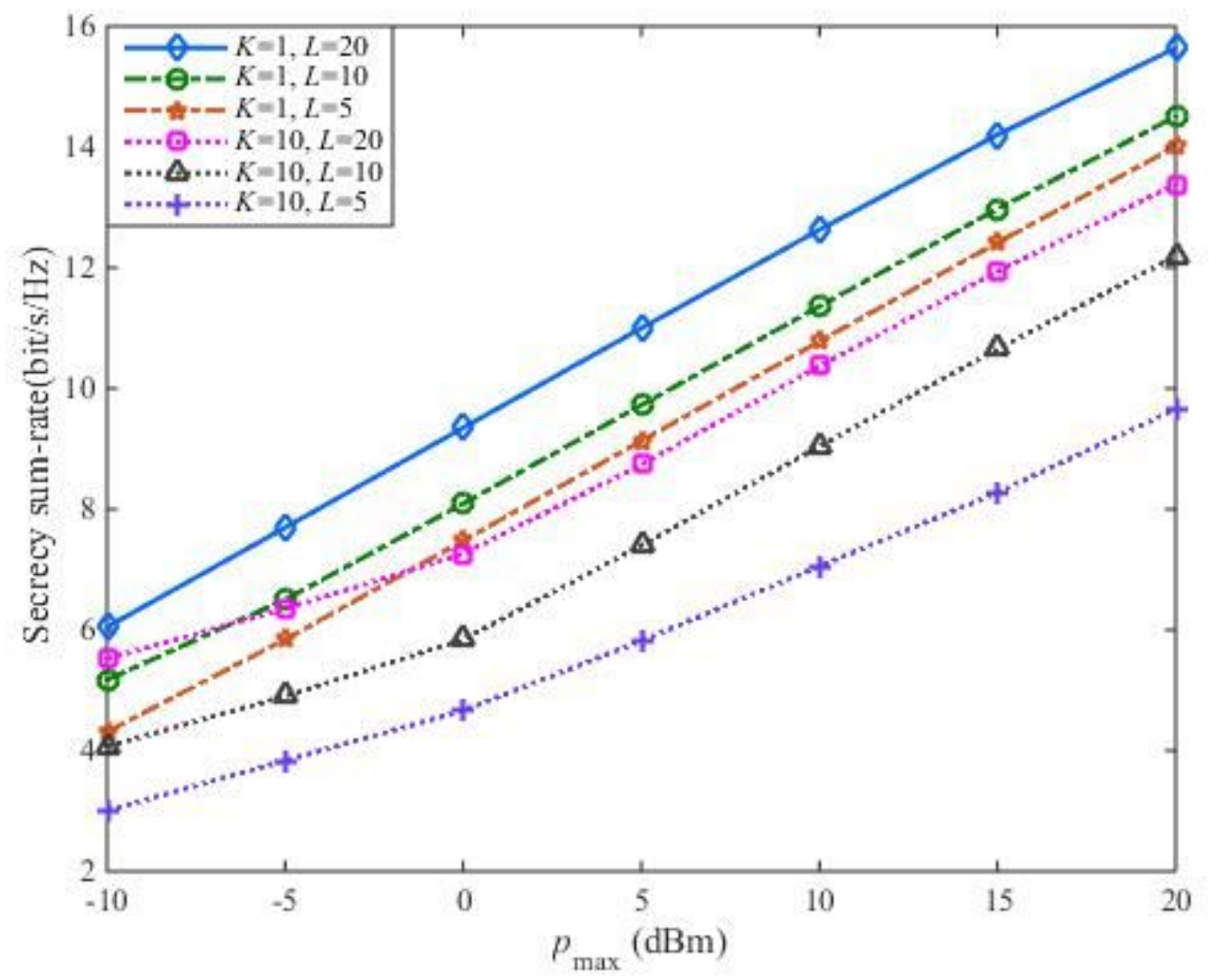


Figure 9

Secrecy sum-rate in different Pmax and $L$ with $P B S=35 \mathrm{dBm}$, $p u=30 \mathrm{dBm}$ and $K=\{1,10\}$ 